\title{
Characterising Spectral Anomalies in Rotating Machine Sensors
}

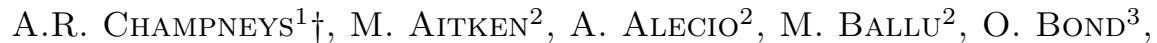 \\ C. Booker ${ }^{2}$, A. Chakraborty ${ }^{4}$, S. Cowley ${ }^{2}$, M. DufF ${ }^{5}$, A. Evans $^{6}$, \\ M. Grinfield ${ }^{7}$, J. Hinch ${ }^{2}$, M. Hoffstetter ${ }^{2}$, K. Kavvadias ${ }^{2}$, \\ G. Khimshashvilli ${ }^{8}$, A. Lefauve ${ }^{2}, \mathrm{~K} . \mathrm{LuOng}^{2}$, S. Machado ${ }^{2}$, \\ G. Mavrogiannis ${ }^{2}$, M. Priddin ${ }^{2}$, B. Piette ${ }^{9}$, C. Sear $^{2}$, \\ M. Tatulea-Codrean ${ }^{2}$, and K. Zahradova ${ }^{10}$ \\ ${ }^{1}$ University of Bristol, UK \\ ${ }^{2}$ University of Cambridge, UK \\ ${ }^{3}$ University of Oxford, UK \\ ${ }^{4}$ University of Cardiff, $U K$ \\ ${ }^{5}$ University of Bath, UK \\ ${ }^{6}$ Smith Institute \\ ${ }^{7}$ University of Strathclyde, UK \\ ${ }^{8}$ Georgia \\ ${ }^{9}$ University of Durham ${ }^{10}$ QMUL, UK
}

(Communicated to MIIR on 8 October 2021)

Study Group: ESGI 145, April 8-12, 2019, Cambridge, UK

Communicated by: S. Cowley

Industrial Partner: Faraday Predictive

Presenter: Geoff Walker, Andy Bates and Will Boulton

Team Members: M. Aitken, University of Cambridge; A. Alecio, University of Cambridge; M. Ballu, University of Cambridge; O. Bond, University of Oxford; C. Booker, University of Cambridge; A. Chakraborty, , University of Cardiff; A.R. Champneys, University of Bristol; S. Cowley, University of Cambridge; M. Duff, University of Bath; A. Evans, Smith Institute, Oxford; M. Grinfield, University of Strathclyde; J. Hinch, University of Cambridge; M. Hoffstetter, University of Cambridge; K. Kavvadias, University of Cambridge; G. Khimshashvilli, Georgia; A. Lefauve, University of Cambridge; K. Luong, University of Cambridge; S. Machado, University of Cambridge; G. Mavrogiannis, University of Cambridge; M. Priddin, University of Cambridge; B. Piette, University of Durham; C. Sear, University of Cambridge; M. TatuleaCodrean, University of Cambridge; K. Zahradova, QMUL

Industrial Sector: Mechanics

Key Words: Rotor Dynamics; Spectral analysis; Nonlinear dynamics

MSC2020 Codes: 37, 93, 81

$\dagger$ Corresponding Author: A.R.Champneys@bristol.ac.uk 


\section{Summary}

Several spectral analysis algorithms have been developed to identify spectral anomalies. A careful literature analysis is undertaken of sources of nonlinearity through bearing distress and other contacts and rubs within rotor dynamics. Several different models for such contact-force or lubrication nonlinearities are synthesised from the literature. These forces are implemented within a simulation of a simple Jeffcott rotor. Reasonable parameter values are identified and results are reproduced that are qualitatively similar to both those observed by Faraday Predictive and seen in the literature. The results enable a tentative hypothesis, using the theory of Arnol'd tongues about how the severity of bearing distress may be reflected in changes to the observed spectrum. Various recommendations are made as to avenues of further work to hone the algorithms, apply spectral analysis to the synthetic simulation data, and to perform more systematic parameter studies. 


\section{Report authors}

Mungo Aitken (Cambridge), Alex Alecio (Cambridge), Marin Ballu (Cambridge), Oliver Bond (Oxford), Cameron Booker (Cambridge), Abhishek Chakraborty (Cardiff), Alan Champneys (Bristol), Stephen Cowley (Cambridge), Margaret Duff (Bath), Alex Evans (Smith Institute), Michael Grinfeld (Strathclyde), John Hinch (Cambridge), Michael Hoffstetter (Cambridge), Konstantin Kavvadias (Cambridge), George Khimshashvilli (Georgia), Adrien Lefauve (Cambridge), Karen Luong (Cambridge), Simon Machado (Cambridge), Gerorge Mavrogiannis (Cambridge), Matthew Priddin (Cambridge), Bernard Piette (Durham), Chris Sear (Cambridge), Maria Tatulea-Codrean (Cambridge), Katerina Zahradova (QMUL)

\section{Executive Summary}

Several spectral analysis algorithms have been developed to identify spectral anomalies. This includes a method to develop a significant 'hump' in a signal compared to a baseline, though low pass filtering and maximisation. An additional algorithm using the Hilbert transform has been developed to identify signs of amplitude modulation of the fundamental frequency, and to identify peaks that can be thus explained. An extension to phase modulation is set out in principle.

A careful literature analysis is undertaken of sources of nonlinearity through bearing distress and other contacts and rubs within rotordynamics. Several different models for such contact-force or lubrication nonlinearities are synthesised from the literature. These forces are implemented within a simulation of a simple Jeffcott rotor. Reasonable parameter values are identified and results are reproduced that are qualitatively similar to both those observed by Faraday Predictive and seen in the literature. The results enable a tentative hypothesis, using the theory of Arnol'd tongues about how the severity of bearing distress may be reflected in changes to the observed spectrum.

Source code is supplied for all the algorithms and simulations implemented. This is research code, which has not been fully tested, and comes with no guarantees of correctness.

Various recommendations are made as to avenues of further work to hone the algorithms, apply spectral analysis to the synthetic simulation data, and to perform more systematic parameter studies. 


\section{Contents}

1 Problem Description $\quad 4$

2 Introduction $\quad 5$

3 Spectral analysis $\quad 6$

3.1 Identifying hump-type anomalies in the signal . . . . . . . . . . . . . . 6

3.2 Hump Size and Location . . . . . . . . . . . . . . . . . . . . . . 8

3.3 Signatures of Modulation and Nonlinear Effects . . . . . . . . . . . . 10

3.3.1 Amplitude Modulation . . . . . . . . . . . . . . . . . 11

3.3.2 Phase Modulation . . . . . . . . . . . . . . . . . . . . . . 12

3.4 Signal Analysis . . . . . . . . . . . . . . . . . . . . . . 19

4 Nonlinear Rotordynamic Modelling $\quad 24$

4.1 Literature review of nonlinear problems in rotodynamics . . . . . . . . . . . 24

4.2 Lubrication theory and bearing modelling . . . . . . . . . . . . . . . 27

4.3 Mechanical models . . . . . . . . . . . . . . . . . . . . . . 28

4.3.1 Jeffcott model for an eccentric rotor . . . . . . . . . . . . . . 28

4.3.2 Solid/solid contact . . . . . . . . . . . . . . . . . 30

4.3.3 Fluid/solid interaction . . . . . . . . . . . . . . . . 31

4.3.4 Simulation model . . . . . . . . . . . . . . . . . 33

4.4 Simulation Results . . . . . . . . . . . . . . . . . . . . . . . . . . . . . . . . . . . 34

4.4 van der Heijden model . . . . . . . . . . . . . . . . . . . . . . . . . . . . . . . . . . . 35

4.4 Lubrication model . . . . . . . . . . . . . . . . . . . 35

5 Main Findings and Recommendations $\quad 40$

$\begin{array}{ll}\text { A Supplied Code } & 46\end{array}$

A.1 Spectral analysis . . . . . . . . . . . . . . . . . . 46

A.2 Rotordyanmics simulation . . . . . . . . . . . . . . . . 48 


\section{Problem Description}

Faraday Predictive is a small Cambridge-based technology company, specializing in the predictive maintenance of rotating industrial equipment such as pumps, fans, compressors, and conveyors. Issues associated with maintenance of this industrial equipment are estimated to cost $\$ 700$ billion pa worldwide, with much of this money being wasted through inappropriate maintenance strategies. Faraday Predictive provides a means of remotely monitoring rotating equipment and diagnosing impending faults. This helps the customer (who might be a water company, for example) maintain their assets in a timely manner and avoid a catastrophic machine failure by scheduling preventative actions well in advance and conversely to avoid doing un-necessary maintenance on a time-schedule when it is not required.

Their technology uses the electric motor driving the equipment as a sensor, by measuring the voltage applied to, and current drawn by, the motor, and identifying subtle distortions in the shape of the current waveform relative to the voltage waveform. These relative distortions, identified by means of a mathematical modelling approach, are expressed as a residual current. The frequency components in this residual current correspond to the characteristic frequencies of the phenomena causing them, which are typically related to deterioration of the equipment, such as bearing wear, belt slippage, internal corrosion, rubbing, misalignment, etc. By matching the observed frequencies against known characteristic frequencies, we are able to identify the likely cause of the distortion, and the amplitude of the signal at this characteristic frequency indicates the severity of the problem.

A range of mathematical techniques are used in each step of this process, and they can be further described and explored if this is of interest, particularly if the initial exam question is solved early in the week. However, the "Exam Question is a specific one in the area of Anomaly Detection:

- How can we reliably detect changes of shape of our spectrum given a noisy signal?

Once this step is firmly established, the subsequent steps in the process can be called into action, and whilst we can describe and discuss these other steps with the group, their effective deployment is all predicated on having detected the anomaly in the first place, so that is where we want the group to focus first. For some failure modes, the problem manifests itself as a simple peak at a particular location on the spectrum, and this can be detected relatively easily. This is not the focus for ESGI. However, some other phenomena show up as a broad hump of signals rather than at a single peak, and so far we do not have a good solution to identifying this sort of shape change.

If the primary "exam question were solved early on during the week, a number of other areas would also be of interest, including:

- What causes these humps, as opposed to peaks?

- What causes signals to show up as sidebands on higher harmonics as opposed to sidebands on the fundamental? In addition, is there a rational basis for weighting the 
significance of one of these higher harmonic peaks as compared to ones on the fundamental?

- Empirically, we see subharmonics (e.g. 1/3, 1/5, 1/7th and sometimes multiples of these, e.g. 2/7th, 3/7ths) of the rotational speed when rubbing friction is present. Can you explain why this should be the case, and why it shows up at the particular frequency in any particular case (e.g. why sometimes $1 / 5$, and other times, $1 / 7$ th)?

\section{Introduction}

This report contains the results obtained during the Study Group and a small amount of subsequent work, tidying up the results and performing further simulations and analyses.

The report is split into two main sections, numbered 3 and 4, which reflects the breakdown of the working groups during the week effectively into two different teams.

The results from the first team, in Sec. 3, concern spectral analysis of the signals provided by Faraday Predictive. First, we describe an ab initio way to extract information on humps in the spectra from the data files supplied. Then we consider signatures of both amplitude and phase modulation and how the Hilbert transform may be used to identify such effects in a given signal.

The second team, who's results are in Sec. 4, considered forward modelling of possible rotor dynamic effects that can lead to different spectral anomalies. A careful literature review finds various candidate effects how various theories from nonlinear dynamical systems theory can be used to explain the resulting dynamics. We also describe mathematical modelling and the implementation within a simple rotor of various rub, contact and lubrication effects. Simulations are provided for several of these models.

Section 5 draws together the findings and offers recommendations for future work. All cited literature is listed, and a separate appendix lists where to find the various pieces of research code, that we have supplied along with this report. 


\section{Spectral analysis}

Analysing a typical signal arising from Faraday Predictive's technology consists of 2 parts. First, identifying the region(s) in the spectrum where an anomaly occurs; and second, interpreting the identified anomaly. In the first subsection below we will describe a method to describe humps of anomalies in spectra. Then, in the following subsection, we will attempt to extract the main features of the spectrum using the concept of amplitude and frequency modulation.

\subsection{Identifying hump-type anomalies in the signal}

The residual current is the difference between the measured current and the normal driving current expected to go through the electric motor to make it, and what it drives, rotate under normal conditions. When a rotating machine develops a problem, the residual current changes. To identify problems, one can try to identify when the residual current differs from what is expected due to already identified modes of vibrations or known resonances.

To do this, one looks at the modulus of the Fourier coefficients of the residual current and compares this to the normal value, referred to as base-line current, to identify anomalies. As the residual signal is typically small compared to the baseline current, one typically analyses the logarithm of the modulus of the Fourier coefficients. Care needs to be taken to identify the 'noise floor', below which amplitude of feature there is no information.

In some instances, the residual has a definite peaks at specific frequencies which are integer multiples, fractions or combinations of the rotation speed of the rotor, or of the $\mathrm{AC}$ frequency of the drive. In other cases, anomalies occur in the amplitude of the residual that exhibit regions or humps of non zero values.

In what follows, we will denote by $X(t)$ the complex time series that we wish to analyse and by $Y(f)$. In particular we will denote by $X_{b}(t)$ the time series of baseline residual current and by $X_{r}(t)$ the time series of the measured residual current Their Fourier transform are then denoted respectively $Y_{r}(f)$ and $Y_{r}(f)$.

To identify anomalies, one must compute the Fourier coefficients of the residual current, subtract from this the Fourier coefficients of the baseline/normal residual and then compute the logarithm of that difference. Because, we are generally interested in the amplitude spectrum, rather than its phase, we thus compute

$$
\log \left(\left|Y_{r}(f)\right|-\left|Y_{b}(f)\right|\right) .
$$

Figure 1 shows a typical output in which there is an anomaly. In particular, note the regions of elevated measured residual compared with the baseline as identified inside the red ellipses. We refer to these as 'humps'.

The first problem then is to find a method to identify humps. We propose a simple algorithm as follows: 


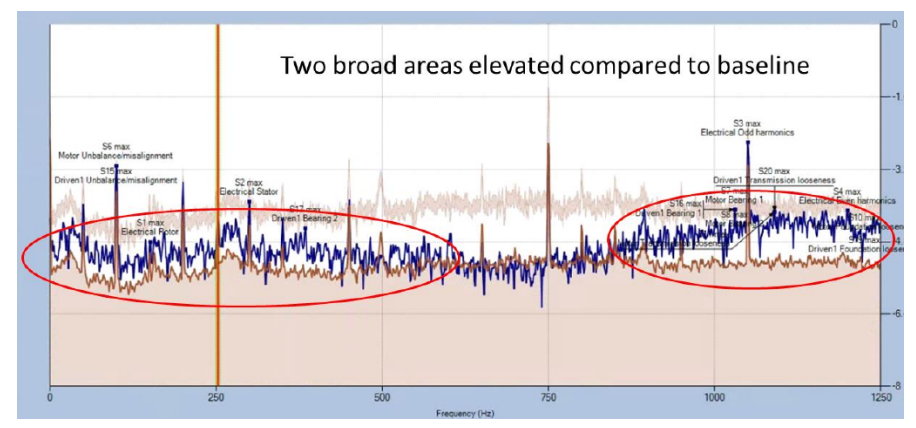

Figure 1: An example of a residual signal (blue) and baseline (orange) as supplied by Faraday Predictive's software.

- Acquire the time series of the residual current $X_{r}(t)$.

- Compute the absolute value of the Fourier coefficients for both the residual and baseline signals: $Y_{r}(t)$ and $Y_{b}(t)$

- Compute the anomalous spectrum defined as

$$
Y_{a}(f)=|| Y_{r}(f)|-| Y_{b}(f)||
$$

- Compute the logarithm of the anomalous spectrum, $\log \left(Y_{a}(f)\right)$, and apply a low pass digital filter to the result. This can be done using a Butterworth filter, or just by computing the Fourier transform of the function, setting to zero all the coefficients above the frequency cut-off and then performing the inverse Fourier transform. The cut-off frequency must be of the order of the inverse of the AC frequency or half of it and one needs to adjust it to select the size of the features that one wants to detect.

One must then compute the exponential of the results to obtain what we call the filtered anomalous spectrum:

$$
Y_{f a}(f)=\exp \left(\text { Filter }_{L P, \nu_{c}}\left(Y_{a}(f)\right) .\right.
$$

Notice that the low-pass cut-off frequency, $\nu_{c}$, is expressed in seconds (inverse of Hertz) but that applying the low-pass filter is not equivalent to truncating the original signal because of the absolute values and logarithms that have been used to compute the anomalous spectrum.

- Compute the $L^{2}$ norm of the filtered anomalous spectrum $Y_{f a}(f)$. This will provide a measure of the anomaly, ignoring anomalies which have a very specific frequency. 


\subsection{Hump Size and Location}

Having identify humps inside spectral data, we can try to determine their size and position. We can do this by performing the following steps:

1. Compute the logarithm of the filtered anomalous signal: $\log \left(Y_{f a}(f)\right)$.

2. For a range of possible hump widths, $w$ compute the convolution of the logarithm of the filtered anomalous signal with a box function, $B_{\omega}(v)$, of width $w$ and area 1 . One can take for example

$$
B_{w}(v)= \begin{cases}\frac{1}{2 w} & |v| \leq w \\ 0 & |v|>w .\end{cases}
$$

We then take the maximum value of the result to be a measure of the hump magnitude which depends on $w$ :

$$
H(w)=\max _{v}\left(\int_{f_{\min }}^{f_{\max }} \log \left(Y_{f a}(u)\right) B_{w}(u-v) d u\right),
$$

where $f_{\min }$ is the lowest frequency of $Y_{f a}(f)$ and $f_{\max }$ a fraction of the sampling $f_{\text {smap }}$ frequency, like $f_{\text {smap }} / 4$ for example.

3. Plot a graph of the hump magnitude measure, $H(w)$, against $w$, the width of the convolution function. The result should be a decreasing function.

4. Find the $w$ that corresponds to the maximum second derivative of the hump magnitude measure. This is your characteristic 'hump' width say $\hat{w}$.

5. Again take the convolution of the logarithm of the filtered anomalous signal, $\log \left(Y_{f a}(f)\right)$, with the box function of width $\hat{w}$. The position of maximum of the result corresponds to the position of the 'hump'.

Results of using the this algorithm are illustrated on three specific data sets provided by Faraday Predictive, referred to as Gena 1, Site A and Site K respectively, are presented in Figures 2-4. The Gena 1 case is an example where there is a definite hump in the data between about 25 and $75 \mathrm{~Hz}$ and this is clearly identified by the algorithm. For the Site A data there is a raised hump at at the low frequency end of the spectrum. The algorithm correctly identifies the right-hand boundary of the hump but has difficulty in deciding where the left-hand (low frequency) end of the hump should be indicated. Finally, the site K example is one in which there is barely any hump. Nevertheless the algorithm identifies a slight hump in the data between about 200 and $600 \mathrm{~Hz}$, which may or may not be significant.

It is useful to make some remarks. Note that this method finds exactly one 'hump'. In the case of multiple humps, this may show up in multiple spikes in the second derivative 

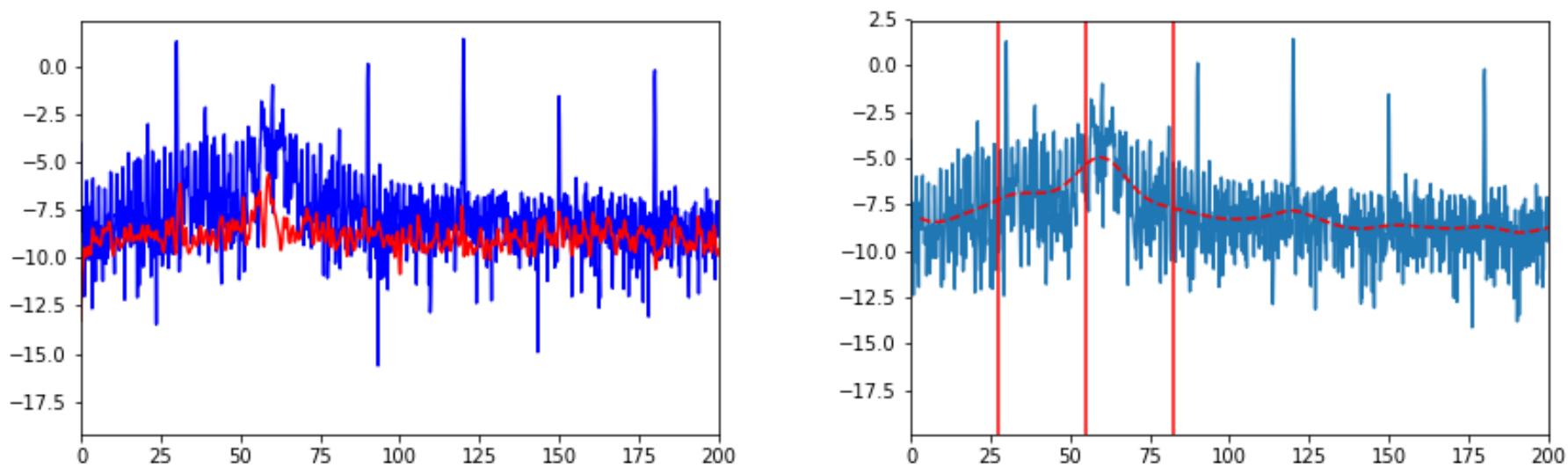

Figure 2: Determination of the hump for the Gena 1 data. (a) Residual (blue) and baseline (red) data. (a) Logarithm of the absolute value of the difference between the residual data and the base line, full data (blue) low pass data with a cut-off frequency of 0.01s (red). The vertical red lines indicates the boundaries of the hump.
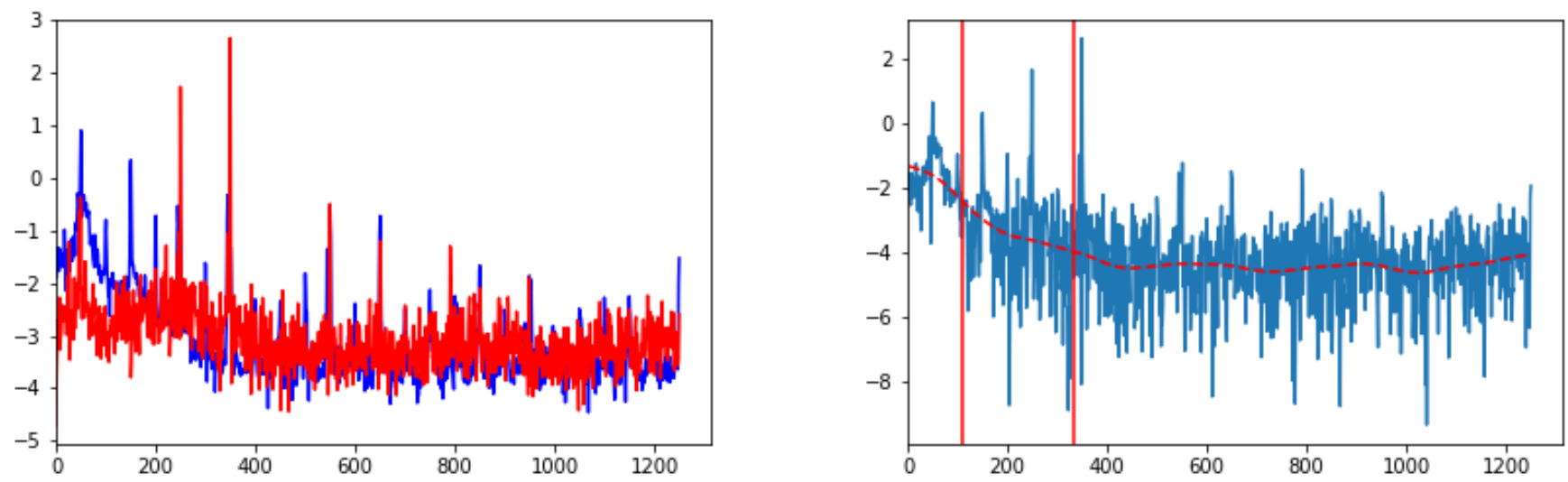

Figure 3: Similar to Fig. 2 but for the Site A data. 

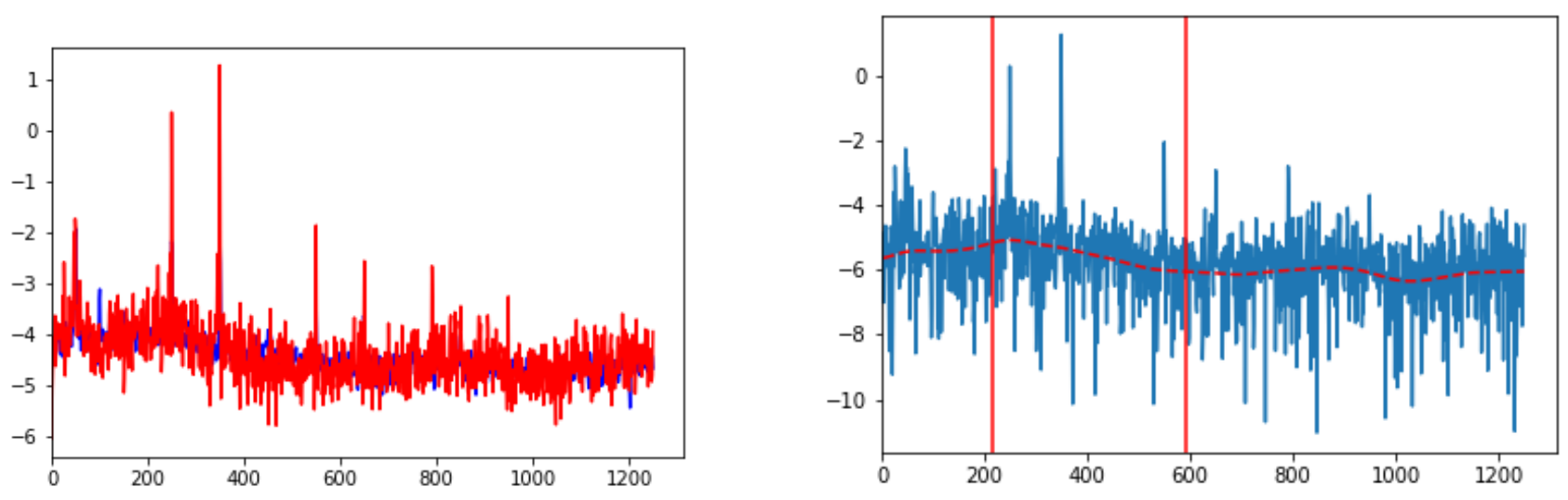

Figure 4: Similar to Fig. 2 but for the Site K data.

graph and the algorithm could be extended to detect these. For each hump found, taking the $L^{2}$-norm of the filtered anomalous signal for the range of the hump will quantify its size, and a significance cut-off could be developed.

Note also that if a baseline signal is not available or not suitable, then it is possible to apply this algorithm directly to a low pass filter of the logarithm of the original signal.

There are some areas that require more rigorous mathematical investigation, these include:

- The choice of the maximum value in point 2 .

- The choice of the second derivative in point 3.

- The choice of a box function for the convolution in point 2. A Gaussian with unit area and varying standard deviation also works on some initial test cases but does not give such sharp peaks in the second derivative of the hump magnitude measure.

\subsection{Signatures of Modulation and Nonlinear Effects}

Some anomalous spectra exhibit specific features that can be understood in terms of modulation theory. Signals that can be adequately described in this way typically involve quasiperiodic effects in which additional frequencies are excited which interact with the fundamental, or weakly nonlinear behaviour, where the signal can be described via a (typically slow) modulation of the amplitude and/or phase of the fundamental. Note that in [1], it is claimed that radial loads on bearings lead to an amplitude modulation of the residual current, whereas non-radial loads lead to a frequency modulation (which is a form of phase 

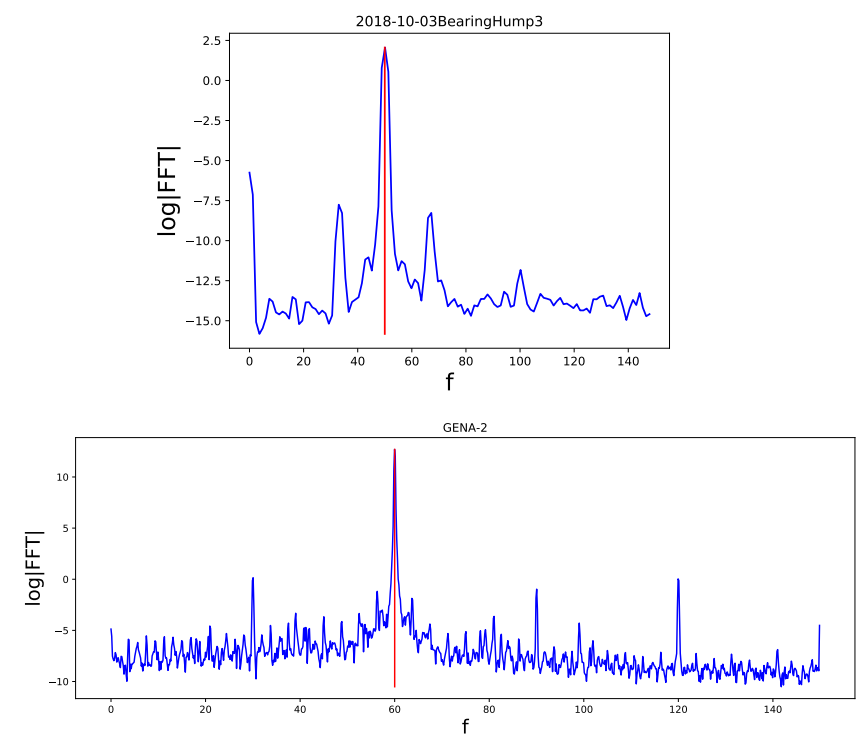

Figure 5: Two example spectra showing possible effects of signal modulation.

modulation), of the residual current. Therefore, we shall try to characterise the hallmarks of both amplitude and phase modulation.

Two examples of measured spectra of residual currents provided by Faraday Predictive are presented in figure 5. Panel (a) shows a signal with an AC frequency of $50 \mathrm{~Hz}$ exhibiting two clear side band peaks around $45 \mathrm{~Hz}$ and $65 \mathrm{~Hz}$. Panel (b) on the other hand, shows a spectrum for a machine that has an $\mathrm{AC}$ frequency of $60 \mathrm{~Hz}$ which has two clear peaks around $30 \mathrm{~Hz}$ and $90 \mathrm{~Hz}$ as well as a series of peaks with decreasing amplitude approximately every $4 H z$.

The aim of this section is to find analytic expressions which depend only on a few parameters to describe the spectra. These functions could then be used as an anstaz for the mechanical modelling of the rotating equipment, or alternatively to compare to a database of values to infer the causes of the observed anomalies.

\subsubsection{Amplitude Modulation}

Amplitude modulation of a sinusoidal AC signal consists of a signal of the form

$$
X(t)=A \sin \left(2 \pi \nu_{A C} t\right)\left(1+B \sin \left(2 \pi \nu_{\text {mod }} t\right)\right) .
$$

Using trigonometric identities this is equal to

$$
X(t)=A \sin \left(2 \pi \nu_{A C} t\right)+\frac{A B}{2}\left[\cos \left(2 \pi\left(\nu_{A C}-\nu_{\text {mod }}\right) t\right)-\cos \left(2 \pi\left(\nu_{A C}+\nu_{\text {mod }}\right) t\right)\right]
$$


and thus the Fourier coefficients correspond to two side-bands located symmetrically around the $A C$ frequency at $\nu_{A C}-\nu_{\text {mod }}$ and $\nu_{A C}+\nu_{\text {mod }}$.

Thus a feature such as observed in Fig. 5(a) corresponds probably to an amplitude modulation of $15 \mathrm{~Hz}$. Similarly, the two larger peaks in Figure 5(b) are likely to correspond to an amplitude modulation of $30 \mathrm{~Hz}$, but the smaller peaks are probably created by something else.

\subsubsection{Phase Modulation}

Phase modulation consists of a signal of the form

$$
X(t)=A \sin \left[2 \pi \nu_{A C} t+\phi(t)\right],
$$

where $\phi(t)$ can be any function, either periodic or not. The simplest case is given by

$$
\left.\phi(t)=A_{1} \sin \left(2 \pi \nu_{\text {mod }} t\right)\right),
$$

for which it can be shown that $X(t)$ can be written as

$$
X(t)=\sum_{n=-\infty}^{\infty} J_{n}(A) \sin \left[2 \pi\left(\nu_{A C}+n \nu_{m o d}\right)\right]
$$

where $J_{N}(A)$ are Bessel functions of the first kind.

Note that equation (7) is the Fourier series of the phase modulated signal, which from the combination of two sinusoidal functions leads to a very rich spectrum. The non-zero frequencies in the spectrum are given by $\nu_{A C}+n \nu_{\text {mod }}$ for each integer $n$.

The typical signature of phase modulation is that the spectrum is symmetric around the central AC frequency. Aside from the large difference in shape, compared to amplitude modulation, the big difference is in the amplitude of the coefficients. When the amplitude of modulation $A$ is small, the side bands decrease rapidly, while for large values of $A$, they remain significant for a larger number of frequencies. This can be seen in the examples of the logarithms of the Fourier coefficients in Fig. ??.

A double phase modulation corresponds to

$$
\left.\phi(t)=A_{1} \sin \left(2 \pi \nu_{\bmod 1} t\right)+A_{2} \sin \left(2 \pi \nu_{\bmod 2} t\right)\right)
$$

and the function $X(t)$ can then be written as

$$
\left.X(t)=\sum_{n_{1}=-\infty}^{\infty} \sum_{n_{2}=-\infty}^{\infty} J_{n_{1}}\left(A_{1}\right) J_{n_{2}}\left(A_{2}\right) \sin \left(2 \pi\left(\nu_{A C}+n_{1} \nu_{\text {mod }_{1}}+n_{2} \nu_{\text {mod }_{2}}\right)\right)\right) .
$$

The results are plot in Fig. 7. We see that double phase modulation has an even richer spectrum; the the non-zero frequencies are given by $\nu_{A C}+n \nu_{m o d, 1}+m \nu_{m o d, 1}$ for all integer 

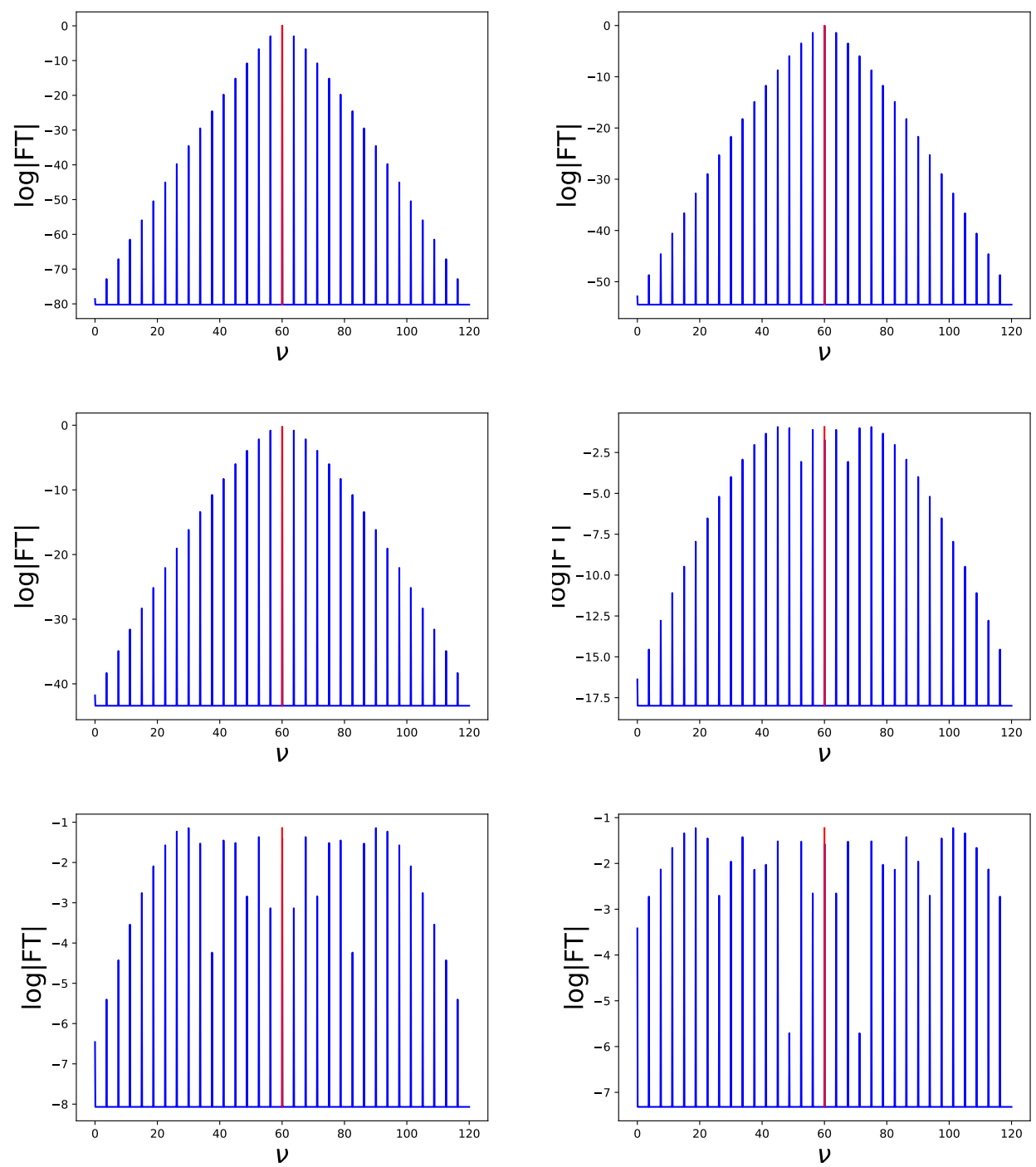

Figure 6: Logarithm of the norm of the Fourier coefficients of a phase modulated signal as given by equation (9) for a carrier of $\nu_{A C}=60 \mathrm{~Hz}$ and $\nu_{\text {mod }}=3.75 \mathrm{~Hz}$. (a) $A=0.1$, (b) $A=0.5$, (c) $A=1$, (d) $A=5$, (e) $A=10$, (f) $A=13$, 

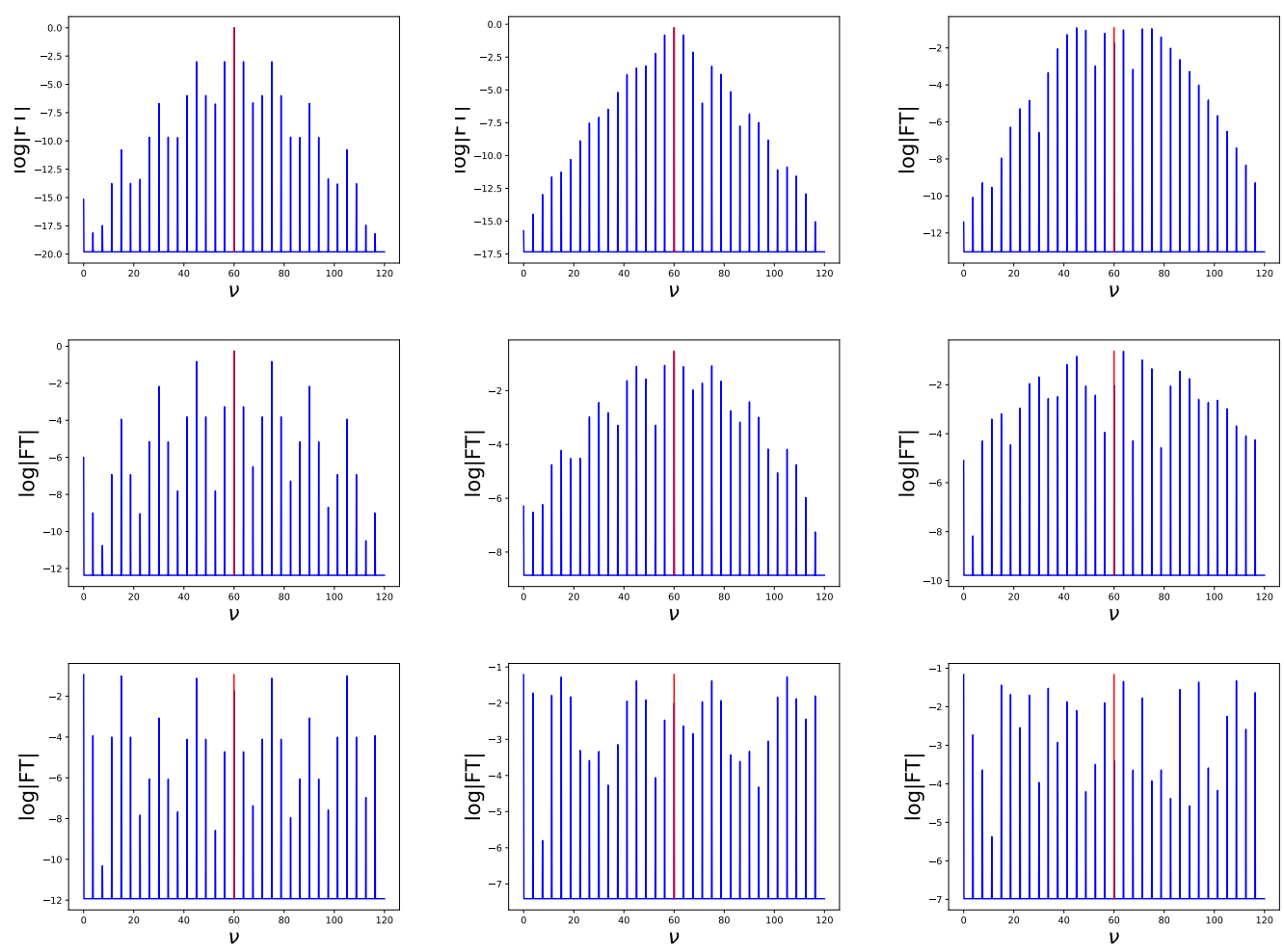

Figure 7: Logarithm of the norm of the Fourier coefficients of a double phase modulated signal (??) for a carrier frequency $\nu_{A C}=60 \mathrm{~Hz}$ and modulation frequencies $\nu_{\text {mod }_{1}}=15 \mathrm{~Hz}$, $\nu_{\text {mod }_{2}}=3.75 H z$. (a) $A_{1}=0.1, A_{2}=0.1$, (b) $A_{1}=0.1, A_{2}=1$, (c) $A_{1}=0.1, A_{2}=5$, (d) $A_{1}=1, A_{2}=0.1$, (e) $A_{1}=1, A_{2}=1$, (f) $A_{1}=1, A_{2}=5$, (j) $A_{1}=5, A_{2}=0.1$, (h) $A_{1}=5$ , $A_{2}=1$, (i) $A_{1}=5, A_{2}=5$,

$n$ and $m$. The double phase modulation also leads to a symmetric spectrum around the $\mathrm{AC}$ frequency but the Fourier coefficients do not decrease as fast as for the single phase modulation.

Several of the data files we have looked at, like for example the one shown on figure 5(b), appear to exhibit spectra that have symmetric or nearly symmetric side bands on either side of the AC frequency. This suggest that these signals are either the results of amplitude modulation, phase modulation or both.

Note that to look more like the measured spectra, rather than compute the exact Fourier coefficients, we can use a different method, which better mirrors the experimental data. Instead we generate the time series mathematically of a time window of $6.5 \mathrm{~s}$ and sampled at $2500 \mathrm{~Hz}$ (to match what is done experimentally). The results are shows in Fig. ?? If we compare figure 8 with figure $6(\mathrm{~d})$ the main difference is that on the sampled figure, the peaks have finite width, and are more rounded; they are smeared out altogether a long way from 


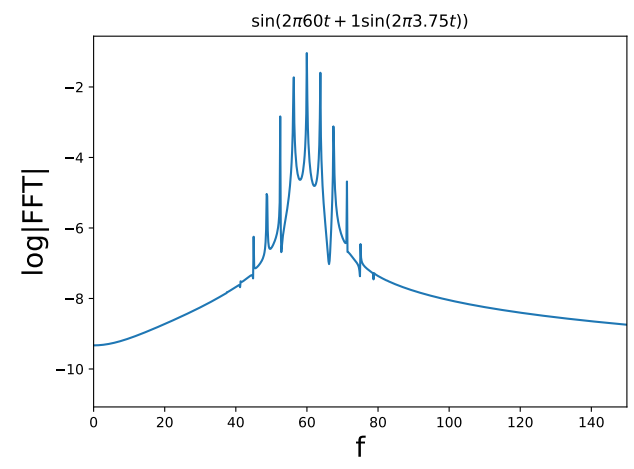

Figure 8: Reconstructed spectrum for a synthetic periodic phase modulation signal constructed by sampling the time series of the signal $\sin (2 \pi 60 t+1 \sin (2 \pi 3.75 t))$.

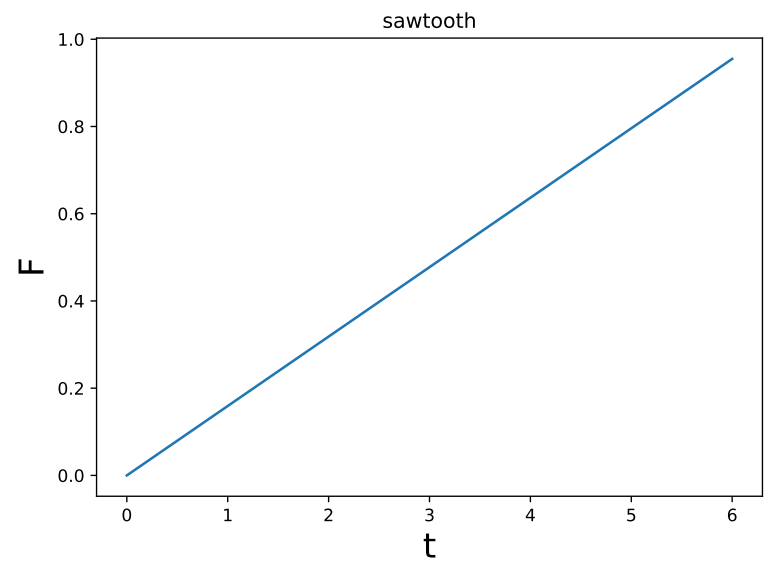

Figure 9: (Half of) a sawtooth function of period $2 \pi$.

the fundamental. The reason is that some of the oscillations have long periods that are not good integer multiple of signal sample. As a result, the spectrum exhibits broader, smoother spikes. These are artifacts of the discretisation. To attenuate them one must take longer samples (increasing the sampling frequency would not help).

Another issue with the spectra we have generated by hand is that the amplitudes appear to decrease more rapidly than the one seen in Fig. 5(b). This suggest that we maybe should use a different phase modulation function.

To investigate this, we have written a python program sawtooth_modulation.py to generate time signals and compute Fourier transform of a sawtooth modulation as shown in Fig. 9 The results are shown in figure 10-12.

The profile of the sawtooth function used for the phase modulation in figure 10-12 is shown on figure 9. 


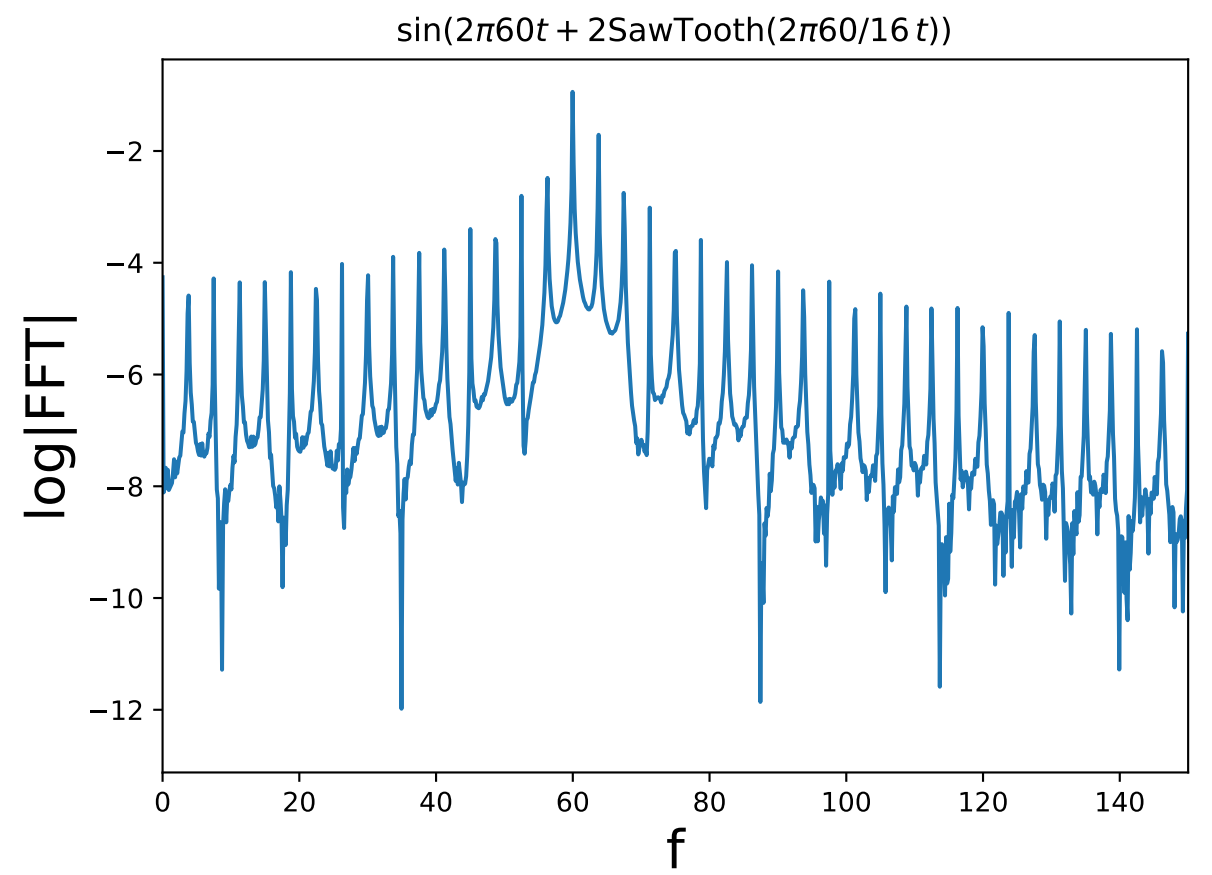

Figure 10: Logarithm of Fourier coefficients for sawtooth phase modulation. $\sin (2 \pi 60 t+$ $2 \operatorname{SawTooth}(2 \pi 60 / 16 t))$ 


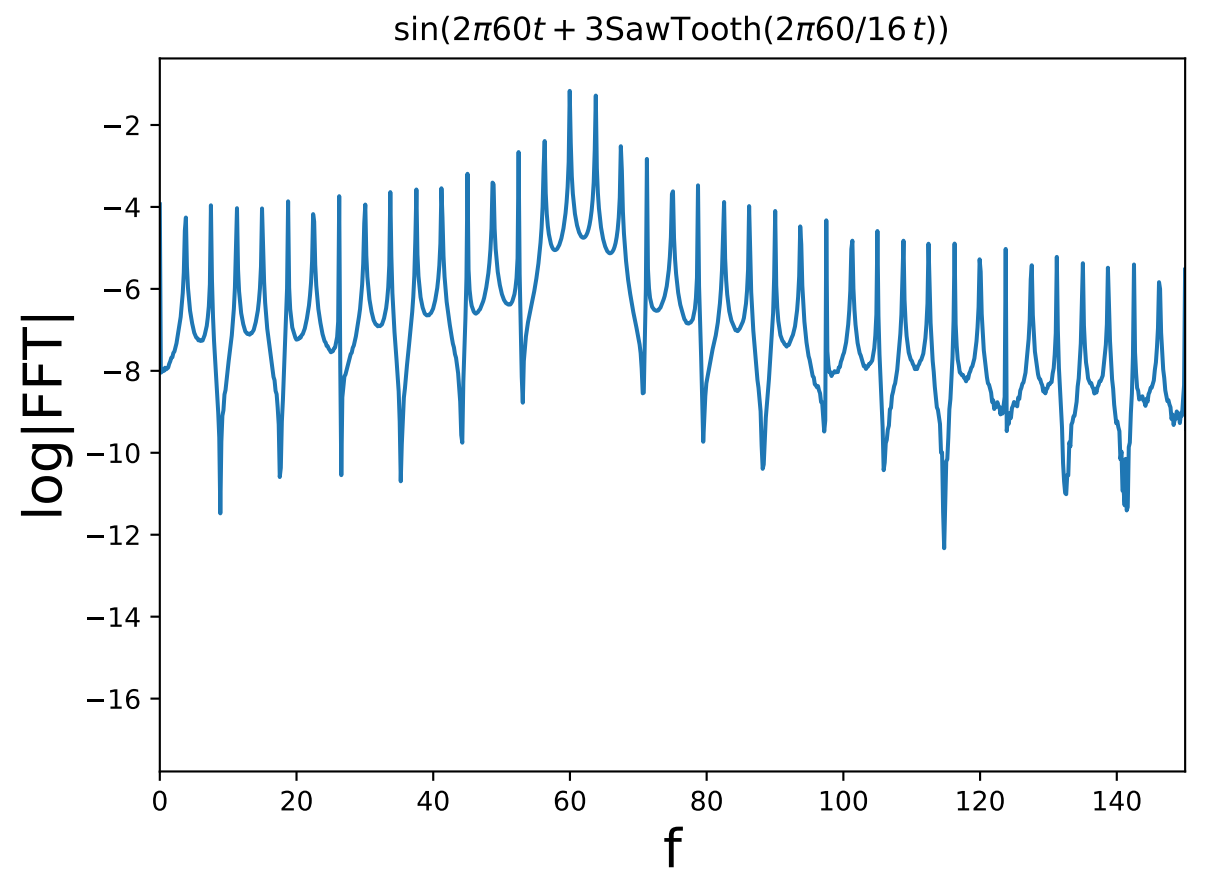

Figure 11: Logarithm of Fourier coefficients for sawtooth phase modulation. $\sin (2 \pi 60 t+$ $3 \operatorname{SawTooth}(2 \pi 60 / 16 t))$ 


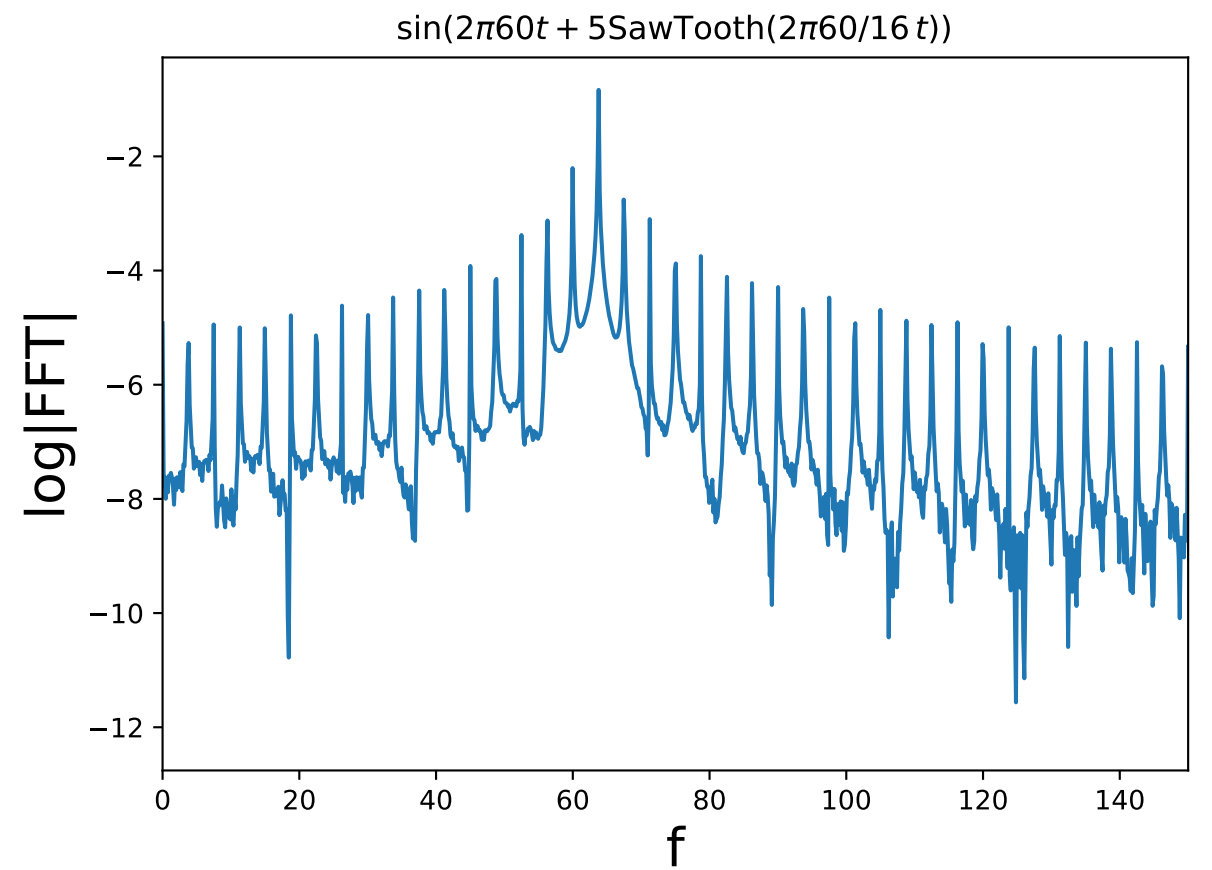

Figure 12: Logarithm of Fourier coefficients for sawtooth phase modulation. $\sin (2 \pi 60 t+$ $5 \operatorname{SawTooth}(2 \pi 60 / 16 t))$ 
The sawtooth modulation does exhibit regular peaks that decay more slowly with frequency than a sinusoidal modulation. This suggests that harmonicly rich functions should be used a phase modulation ansatz to try to characterise broader regions of significant spectra. Such non-periodic slow modulation of a signal are hallmarks of a weak nonlinearity in the system, because the signal cannot be approximately captured by a finite combinations of sine waves.

Nevertheless, dense regions of non zero frequencies, which we referred to as hump above, are more likely to be hallmarks for strong nonlinear behaviour. In particular, it is known that chaotic behaviour leads to broad band spectra. Possible mechanisms for the onset of such behaviour will form the subject of Sec. 4.

A good way to test whether a signal is chaotic or not though is to compare time series computed over relatively short times and over longer times. If the signal is truly chaotic rather than a modulated sine wave, then the spectrum should become denser for longer time series, whereas a quasi-periodic signal will develop more pronounced isolated peaks.

\subsection{Signal Analysis}

We shall now explain how one can extract we try to extract amplitude (and in principle) phase modulation of the signal using the Hilbert transform.

The Hilbert transform is defined as

$$
H(X(t))=\frac{1}{\pi} \int_{-\infty}^{\infty} \frac{X(t)}{\tau-t} d \tau .
$$

It can be shown that the Hilbert transform of the trigonometric functions are the following:

$$
\begin{aligned}
& H(\sin (2 \pi \nu t))=-\cos (2 \pi \nu t) \\
& H(\cos (2 \pi \nu t))=\sin (2 \pi \nu t)
\end{aligned}
$$

where $\nu$ is assumed to be positive. One also defines the $Z$ transform of a real function $X(t)$ as follows

$$
Z(X(t))=X(t)+i H(X(t)) .
$$

If $X(t)=A \cos (2 \pi \nu t)$, then $Z(X(t))=A \exp (2 \pi i \nu t)$, and if $X=A \sin (2 \pi \nu t)$, then $Z(X(t))=$ $-i A \exp (2 \pi i \nu t)$, So, if one decomposes any signal $X(t)$ as a series,

$$
X(t)=\sum_{n}\left(a_{n} \cos (2 \pi n \nu t)+b_{n} \sin (2 \pi n \nu t)\right),
$$




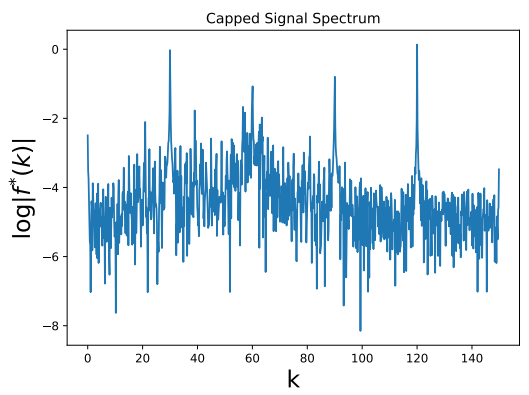

Figure 13: The spectrum of the example signal GenA-1_ia

one can see that the $Z$ transform provides an analytic continuation of the function $X(t)$. Specifically, we can write

$$
\begin{aligned}
Z(X(t)) & =\sum_{n}\left(a_{n}-i b_{n}\right) \exp (2 \pi i n \nu t) \\
& =A(t) \exp (i 2 \pi \phi(t))
\end{aligned}
$$

where $A(t)$ and $\phi(t)$ are real functions.

Based on the observations above, if we suppose that a signal is of the form

$$
X(t)=A(t) \cos (2 \pi \phi(t)),
$$

and if we compute $Z(X(t))$, then the amplitude of the signal $X(t)$ is given by $A(t)=$ $|Z(X(t))|$ and the frequency can be extracted from the argument of $Z(X(t))$. Note that within python, the hilbert transform of the scipy. signal module actually computes the $Z$ transform of the signal.

To compute the amplitude of the signal, we have produced a program AM_resonstruction_v2.py to compute the modulus of the $Z$ transform of the signal. We then compute the Fourier coefficients of that function and remove all the coefficients that are below a threshold $h$ before reconstructing the amplitude function.

We have use the program AM_resonstruction_v2.py to analyse the example time series data GenA-1 provided by Faraday Predictive.

Figure 13 shows the spectrum of the GenA-1 residual current. One should emphasize that for this analysis, we must start from the time signal and that we are unable to subtract any baseline. This is because there is no way to know what the phase of the background signal might be. So one must analyse a typical residual current first and then compare the result to an anomalous residual current.

Figure 15(a) shows the filtered spectrum of the extracted amplitude modulation for a threshold value of 0.15 . Only 4 peaks have been kept: $30 \mathrm{~Hz}, 60 \mathrm{~Hz}, 90 \mathrm{~Hz}$ and $120 \mathrm{~Hz}$. Figure 15(b) shows the reconstructed time series over 6.5s. Once can see that it is modulated and that the system is not completely steady. 


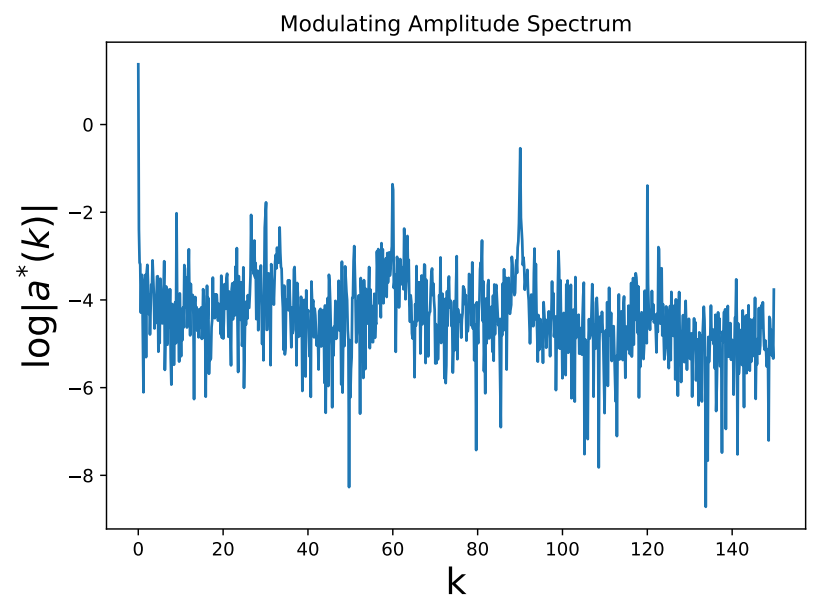

Figure 14: The computed amplitude modulation of the GenA-1_ia example.
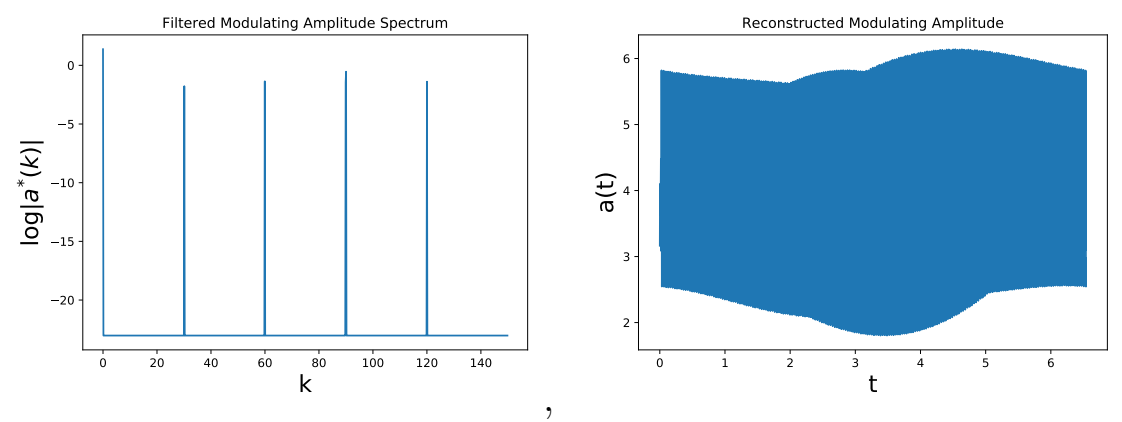

Figure 15: The filtered amplitude modulation of GenA-1_ia, using 0.15 as the threshold. (a) The peaks retained; (b) the reconstructued signal. 

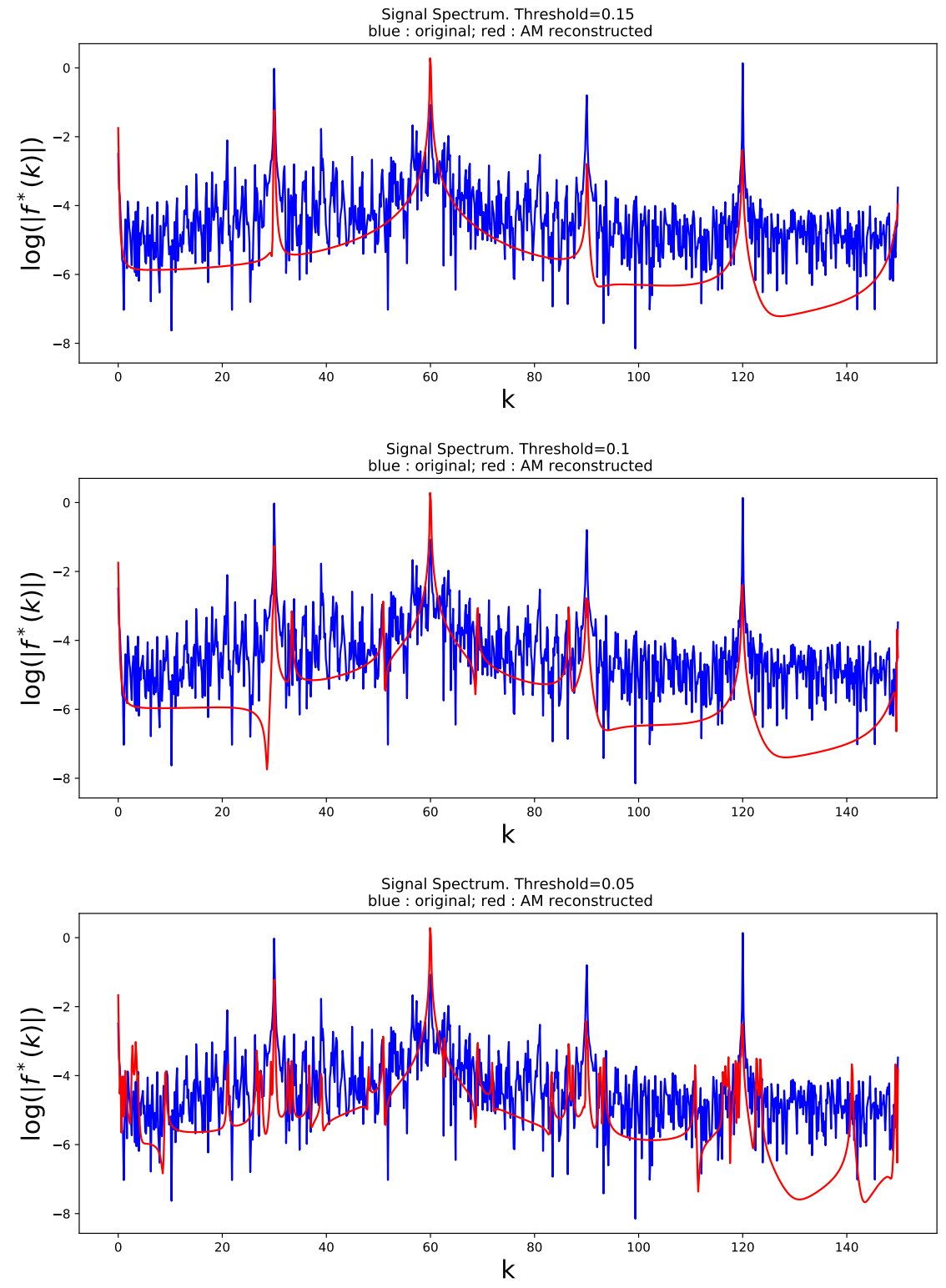

Figure 16: The GenA-1_ia original spectrum (blue) and reconstructed spectrum (red) for the threshold (a) 0.15, (b) 0.1, (c) 0.05 . 
Figure 16 compares the reconstructed amplitude modulated signal with the original one for 3 threshold values: $0.15,0.1$ and 0.05 . The 4 th largest peaks looks like evidence of amplitude modulation while the others do not seem to be so. To explain the additional complexity of the signal, one should perhaps attempt to parametrise the signal using phase modulation.

It is clear that the peaks should be matched better, but to achieve this, one would have to extract the phase and the amplitude modulation simultaneously and reconstruct the time series from their filtered versions. The implementation of such analysis automatically is left for future work.

One point that it is interesting to note from the raw data in Fig. 16 is that there is a small hill around the AC frequency $(60 \mathrm{~Hz})$ and this is caused by the low frequency modulations with frequencies between 1 and $2 \mathrm{~Hz}$. As these corresponds to large period, they do not always fit well within the $6.5 \mathrm{~s}$ second windows used for sampling. To avoid these hills one should use longer sampling windows. Notice that if the signal contains some oscillations of even lower frequencies, the problem could persist as these frequencies will have a similar effect, but only if their amplitudes are large enough. Another reason to recommend larger samples is that any evidence of chaotic dynamics would be likely only revealed by comparing evolution of the spectrum over a longer time span. 


\section{Nonlinear Rotordynamic Modelling}

Since the invention of the wheel, rotating machines have been a central part of human life. In fact, the dynamics of spinning rotors underlies just about every man made machine, from power generators, internal combustion, electrical and aero engines, to every day tools such as drills, lathes and mills, to critical mechanical components of larger plants and assemblies, such as fans, pumps and compressors. The monitoring and servicing of such rotors therefore remains a critical part of maintaining the performance of just about every mechanical system that we take for granted in everyday life.

Before developing a mathematical model to investigate the possible sources of nonlinear behaviour of a spinning rotor, it is useful to conduct a survey of the relevant literature.

\subsection{Literature review of nonlinear problems in rotodynamics}

Rotating machines are unlike other mechanical vibration systems because of the large amount of rotational kinetic energy that can be transferred into lateral or torsional vibration modes whenever the rotor is out of balance. Reducing these vibrations is often the main goal of the design and control and maintenance of rotating machines.

Extensive recent research has been carried out on exploring, both experimentally and through mathematical models, the nonlinear vibrational dynamics of rotating machines, see e.g. $[2,3,4,5,6]$ and references therein for recent developments. The typical mechanisms that allows energy transference to vibration modes from the rotating mass is the presence of centrifugal forces due to out-of-balance or out-of-alignment eccentricity. These abnomormalities may arise due to manufacturing and assembling deficiencies, or due to operational conditions and wear. Finite amplitude behaviour is typically then highly sensitive to resonance between vibrational modes and the rotor drivespeed, and is exacerbated by the presence of various kinds of nonlinearity.

Much information, including lots of experimental results can be found in the book by Muszynska [7]. She has found many models that demonstrate subharmonic response.

Perhaps the most common causes of nonlinear behaviour in any rotor are a wide variety of effects due to the dynamics of the bearings; see for example [8]. Here the specific source of bearing nonlinearity can vary from squeeze film effects [9], so-called dry whip of journal bearings [10] to magnetic bearing singularity effects [11].

Another major class of nonlinearity in rotor dynamics is geometric nonlinearity of varies kinds, see e.g. [12]. Also Luczko [13], included the so-called von-Karman nonlinearity, curvature effects, and large displacement gyroscopic and shear effects, and [14] et al analysed the effect of a cracked shaft on the onset of a whirl instability.

A more violent source of geometric nonlinear behaviour is the presence of free masses that are able to rotate in order to form an automatic dynamic balancing mechanism, see $[15,16,17]$ and references therein for theoretical and experimental results. Basically all of 
these systems, which are able to balance a rotor beyond its fundamental resonance, suffer from the possibility of mode-locking into large-amplitude sub-harmonic whirling limit cycles, similar to those described by van der Heijden [18] for a Jeffcott rotor.

This latter work [18] was one of the first to consider rotor-stator contact from the point of view of nonlinear dynamics. This was inspired by the problem of rotating flexible shafts in drillstrings, given the possibility of borehole interaction; see also [19]. Such quasi-periodic motion and even chaotic motion has also been observed by a number of other authors, see for example [20].

Probably the most extreme and most damaging form of nonlinear behaviour in rotordynamic systems is due to rotor-stator contact, see e.g. [21, 20]. Such problems often occur due to interactions when overhung rotors, such as aircraft engines come into contact with socalled snubber rings that are designed to limit vibration amplitude [22, 23]. Such problems are also relevant for micro-electro-mechanical system (MEMS) rotors [24].

The nonlinear behaviour associated with rotor-stator contact can largely be characterised as being due to one of two effects; namely sustained frictional contact or 'rub', and intermittent contact or 'bouncing.' Studies focused on rub include [25, 26, ?] which have shown that both forward and backward permanent-in-contact whirl motion is possible depending on whether the rotor slips or sticks around the stator. Under rub conditions, torsional effects become important [27] and can give rise to Hopf bifurcations and stick-slip behaviour [28].

In contrast, the dynamics associated with bouncing is potentially more violent than pure rub. Bouncing motion has also proved more problematical to analyse, being fundamentally unsteady; nevertheless see [?, 29] for an analysis using the theory of piecewise-smooth dynamical systems. The work of Zilli et al [6] was one of the fist studies to propose a theory, backed by simulations for the onset of asynchronous bouncing orbits with numerical They proposed a synchronisity condition, which was later shown by Shaw et al [30] to be equivalent to an internal resonance condition when viewed in a rotating frame

See for example the Fig. 17 from the paper by Ma et al [31] which compares the results from an impact and rub model with some of Muszynska's [7] experimental results. Both the model and the experiments show clear signs of sub-harmonic phase-locked motion that coexists with smooth, non-contacting behaviour for rotor speeds that are beyond the fundamental resonance speed of the rotor.

Another insightful set of experimental results were obtained by von Groll and Ewins [?]. They again saw significant subharmonic responses due to rotor rubbing, which they were able to reproduce in a model which incorporated Hertzian contact and Coulomb fricion. By varying the rotation speed they were able to find short intervals of speed at which there was frequency locking into subharmonics whose ratio to the drive frequency had integer denominator. Specific frequency ratios of $6 / 11,12 / 13,1 / 2,15 / 32$ were found. They commented that [?]

The mechanism that is seen to be at work here might be of importance for certain contact scenarios: The rotor and stator structures "find a way" to respond at 


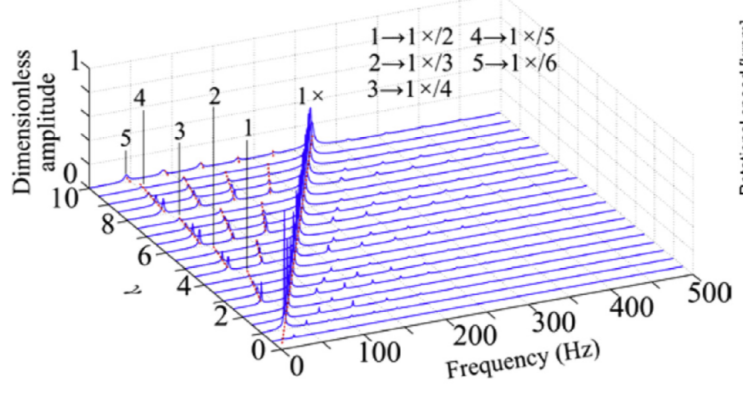

(a)

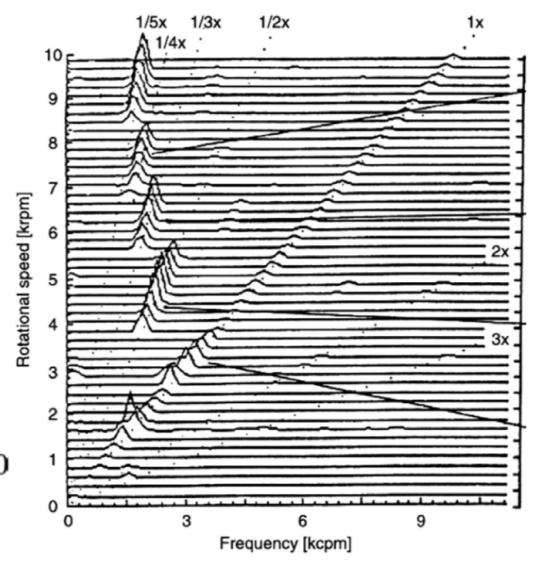

(b)

Figure 17: Comparison between simulation and experimental results for a stator rubbing problem from the paper [31]. The experimental results are taken from [7]

their "combined resonance frequency", even though it might be much lower than the excitation frequency, and even very low sub-harmonics of the rotor speed can potentially feature strongly in the response spectrum

Occasional, partial rubbing and impact was also investigated theoretically and numerically by Goldman and Muszynska [32]. They concluded that

In the wide frequency range, the numerical results showed the sequence of subharmonic and chaotic regimes strongly correlated with the number of impacts per oscillation. For the lower orders of harmonic and subharmonic vibrations, the dominant, with the widest frequency ranges, are one-impacting-per-oscillation periodic regimes. All other regimes represent bifurcation and transition between the latter. The same is true for $1 / 3 x$ oscillation, but the tendency of the multiimpacting and chaotic regimes to widen their frequency band was noted. This indicates an increasing intensity of the chaotic motion at higher frequencies. Another worthmentioning result presented in this paper is an insight into the influence of mixed viscous/dry friction forces at the contact, as well as the influence of rotor anisotropy on the rubrelated responses.

This phenomenon of subharmonic frequency locking is reminiscent of the phenomenon of Arnol'd tongues in input frequency ratio versus amplitude plots. A good explanation of this effect can be found in the Scholarpedia page on "Synchronization"

http://www. scholarpedia.org/article/Synchronization 


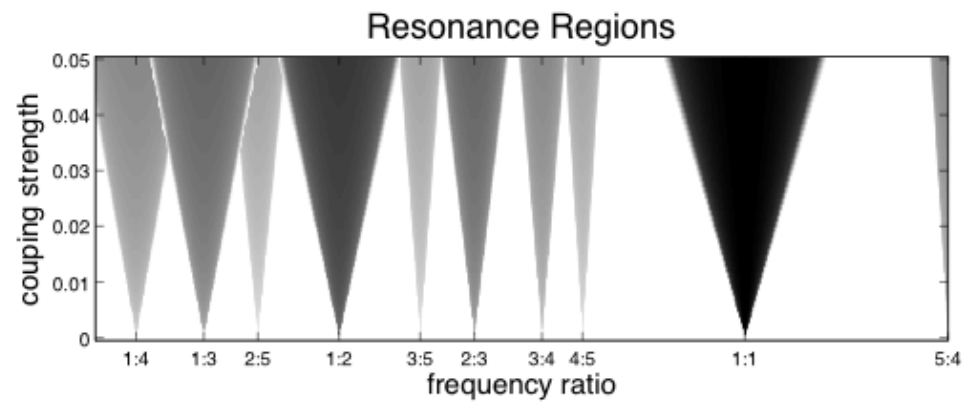

Figure 18: Arnol'd tongues. See text for details

An example of this general phenomenon is illustrated in Fig. 18. Here each tongue represents a parameter region in which there is frequency locking (the shaded regions in the figure). That is, despite the frequency ratio between two natural frequencies in the system being a general irrational number, one finds periodic behaviour which repeats are $n$ cycles of one frequency and $m$ of the other. This is called an $m: n$ mode-locked or quasi-periodic behaviour which appear on a spectral plot as a sequence of peaks at the fundamental and at multiples of $1 / n$th of this frequency.

Arnol'd tongue type mode-locking was also found in a highly simplified model of rotor dynamics with contact by van der Heijden [18].

Recently, a number of theories have been produced for the onset of bouncing type motion in rotor-dynamics due to partial contact, see [6,30]. Those papers refer to internal resonance between forward and backwards whirling modes as being responsible for instability.

Note how most of the experimental and numerical studies in the literature use the rotation speed as a bifurcation parameter, but Faraday Predictive are interested in cases where the rotor is run at a fixed rotation speed but somehow deteriorates over time. We need to understand what such a deterioration parameter may be. We assume that it is due to rubbing, and perhaps due to either degradation of the bearing or increased eccentricity of the rotor.

\subsection{Lubrication theory and bearing modelling}

It is well known that bearings can be a significant source of nonlinearity in rotor dynamics, both nonsmooth rub and contact type effects, as well as smooth nonlinearity due to squeezefilm lubrication effects.

The effects of magnetic bearings have been modelled in the work of Keogh and others, see $[?, 11,29]$ and refernces therein. Ball bearings can naturally introduce different frequencies into the spectrum due to frequencies associated with the ball passing frequencies; see, for example [33]. 
For this report, we have focused on the behaviour of journal bearings. These are lubricated sealed units in which the lubricant is constantly filtered and refilled [34]. Many potential nonlinear effects have been identified in the literature, including the possibility of wear causing unfiltered particles to appear within the lubricant, causing periodic contacts at less than the rotation frequency. See e.g. $[35,36]$

\subsection{Mechanical models}

We shall develop a number of simple mathematical models capable of showing nonlinear behaviour due to rotor-stator contact. General theory of the derivation and analysis of multiple-degree of freedom rotor dynamic models is available in the textbook [37]. Here though we shall focus on the simplest possible Jeffcott model of an eccentric rotors and consider the effects of different types of bearing-rub nonlinearity behaviour. We shall ignore specific bearing models, (such as ball or magnetic bearings) but assume a generic journal bearing which can be modelled either by solid-solid interaction or through lubrication theory.

\subsubsection{Jeffcott model for an eccentric rotor}

The system we have in mind is sketched on the left of figure 19. The stator, in yellow, as radius $R_{1}$, while the rotor, in blue, has radius $R_{2}$, and rotates at constant speed $\Omega$. The gap between the two is $h=R_{2}-R_{1}>0$, it is generally very thin (much thinner than shown here for clarity), and it is typically filled with a lubricant (viscous oil). We denote the horizontal coordinate by $x$ and the vertical coordinate by $y$.

In general, all rotors are eccentric, as sketch on the right of figure 19: their centre of rotation is not coincident with the center of the stator. This is due to manufacturing or operating imperfections, typically because the centre of rotation $\mathbf{r}_{\mathbf{r}}$ is not coicident with the centre of mass $\mathbf{r}_{\mathbf{m}}=\mathbf{r}_{\mathbf{r}}+\varepsilon$ ( $\varepsilon$ is the eccentricity). This results in an eccentric force proportional to the magnitude $\epsilon$ (norm of the vector $\varepsilon$ ) which prevents the centre of rotation from sitting in the middle of the stator (in other words, $\mathbf{r}_{\mathbf{r}} \neq(0,0)$, and we use the notation $\mathbf{r}_{\mathbf{r}}=r \exp i \psi=(r \cos \psi, r \sin \psi)$ to describe it $)$.

To describe the motion of the rotor, we need to develop the model sketched on the left of figure 20 for the center of mass $\mathbf{r}_{\mathbf{m}}$ whose coordinate we call $(x, y)$. To do this, we use Newton's law:

$$
m\left(\begin{array}{l}
\ddot{x} \\
\ddot{y}
\end{array}\right)=\sum \mathbf{F}_{\text {external }}
$$

which means that the mass of the rotor $m$ multiplied by the acceleration of the centre of mass of the rotor equals all the external forces applied to it. These forces are:

- linear stiffness and damping (in red) with constant stiffness coefficient $k$ and damping coefficient $c$, these are typical in any oscillator and reflect the fact that the fluid in the bearing will both oppose the motion of the rotor and dissipate some of its energy.

Page 28 of 48 

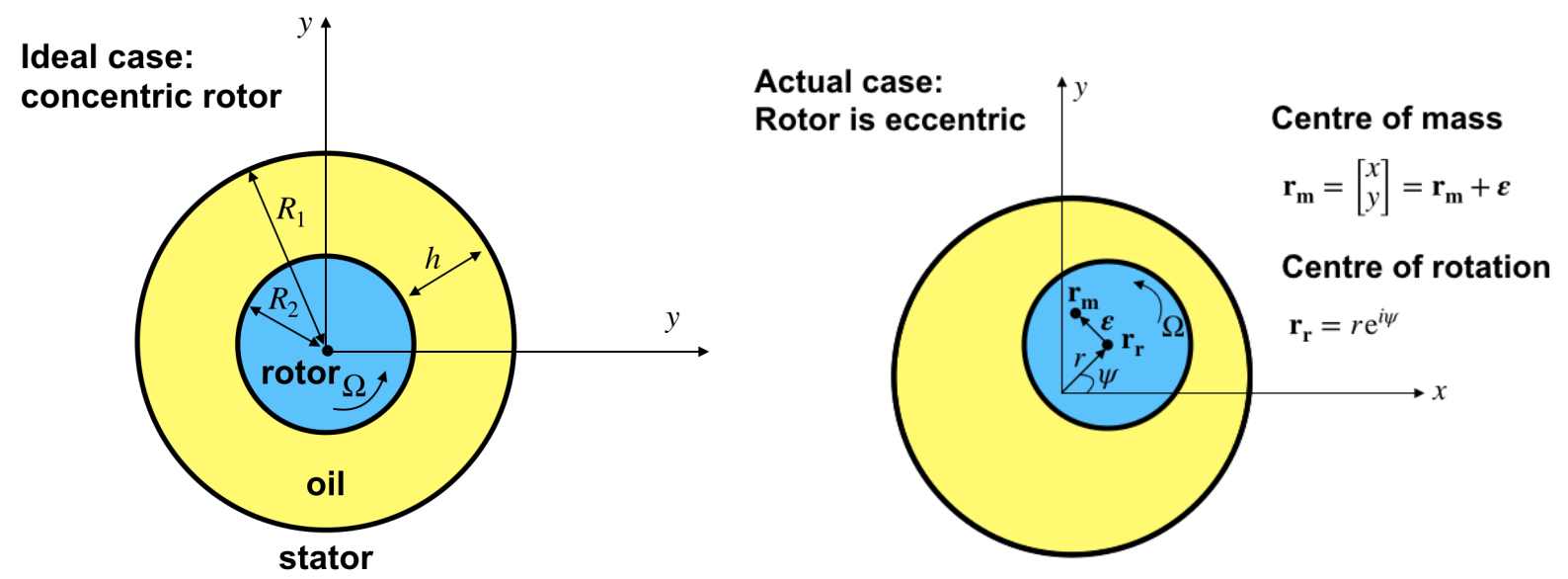

Figure 19: On the left, ideal case of an concentric rotor, primarily used to introduce the notation of radii, gap and rotation speed $R_{1}, R_{2}, h, \Omega$. On the right, the actual case of interest of an eccentric rotor. We introduce further notation: the centre of mass vector $\mathbf{r}_{\mathbf{m}}$, and centre of rotation $\mathbf{r}_{\mathbf{r}}$, separated by the eccentricity vector $\varepsilon$.
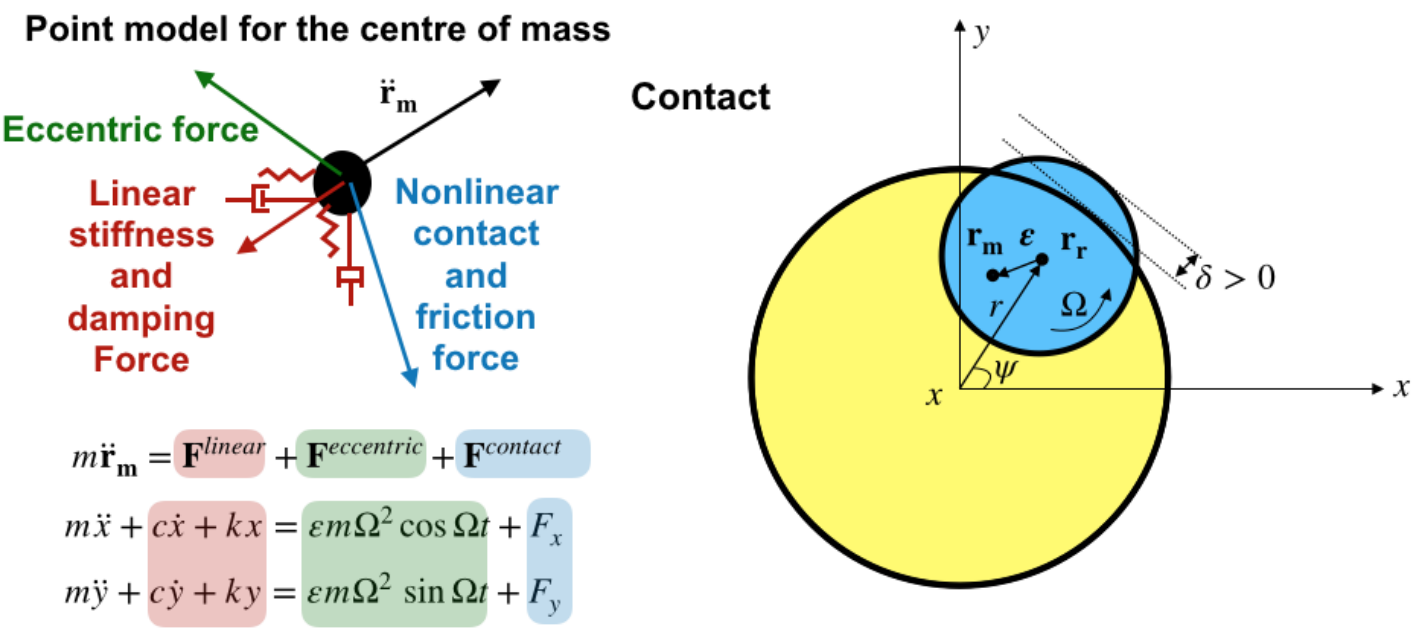

Figure 20: On the left, dynamical two-degrees-of-freedom model for using Newton's law and three types of forces (red, green, blue). On the right, sketch and notation for the solid-solid contact model. 
- eccentric force (in green), whose expression depends on eccentricity, mass, and rotation speed, it is the term that gives energy to these oscillations

- any other nonlinear force (in blue)

In order to generate subharmonics, nonlinearities are necessary (i.e. the force must be nonlinear in $x, y, \dot{x}$, or $\dot{y}$ ): they will be provided by this last 'blue' force only. There areat least two candidates for nonlinearities:

1. solid contact between the rotor and the stator,

2. and the fluid/solid interaction due to the complex fluid motions within the lubricant

In the next two sections we look at how these two types of forces have been modelled in the literature and propose some extensions/simplifications.

\subsubsection{Solid/solid contact}

On the right of figure 20 we show our notation for the solid/solid contact force to harmonise the notation of various models found in several papers. We assume that the rotor penetrates slightly into the stator (usually made of a very soft metal) by a depth $\delta>0$ (but very small):

$$
\begin{aligned}
& \delta=r-h \quad \text { when } \quad r>h \\
& \delta=0 \text { when } r<h .
\end{aligned}
$$

This equation above embodies a strong nonlinearity by the fact that the force can turn on and off depending on $r-h$.

In table 1 we give a list of models for the forces tangential and normal to the contact points based on various powers of $\delta$ and the contact speed $\dot{\delta}$ (the last row being the fluid model discussed in the next section 4.3.3):

In order to obtain $F_{x}$ and $F_{y}$ (horizontal and vertical forces) from $F_{n}$ and $F_{t}$, we use the following equations:

$$
\begin{aligned}
& F_{x}=-F_{n} \cos \psi+F_{t} \sin \psi \\
& F_{y}=-F_{n} \sin \psi-F_{t} \cos \psi
\end{aligned}
$$

where $\psi$ is the contact angle (see figures 19-20) given by:

$$
\begin{aligned}
\cos \psi & =\frac{x-\varepsilon \cos \Omega t}{r} \\
\sin \psi & =\frac{y-\varepsilon \sin \Omega t}{r}
\end{aligned}
$$




\begin{tabular}{|c|c|c|}
\hline Model & $F_{n}$ & $F_{t}$ \\
\hline $\begin{array}{l}\text { van der Heijden }(1995)[18] \\
\quad \text { (also assume } k=0)\end{array}$ & $\begin{array}{c}k_{c} \delta \\
\text { with } \varepsilon=0 \text { in def. of } \delta\end{array}$ & 0 \\
\hline Hua et al. (2015) [38] & $k_{c} \delta+\xi \delta^{2}$ & $\begin{array}{c}\mu f_{n} \\
\text { (Coulomb dry friction) }\end{array}$ \\
\hline von Groll \& Ewins (2002) [22] & $\begin{array}{c}k_{c} \delta^{3 / 2}\left(1+\frac{3}{2} c_{c} \dot{\delta}\right) \\
(\text { Hertzian contact) }\end{array}$ & $\mu f_{n}$ \\
\hline Simplified Groll & $\begin{array}{c}k_{c} \delta+c_{c} \dot{\delta} \\
\text { (not Hertzian) }\end{array}$ & $\mu f_{n}$ \\
\hline Muszynska (2005) [7] & $\begin{array}{c}k_{c} r \text { only if } \Omega t \in\left[T_{0}, T_{1}\right] \bmod 2 \pi \\
(\text { partial rub at every rotation) }\end{array}$ & $\mu f_{n}$ \\
\hline Modified Muszynska & $\begin{array}{c}k_{c}(\delta-a) \text { only if } \psi \in\left[\psi_{0}, \psi_{1}\right] \\
\text { (obstacle } a \text { sticking out of stator) }\end{array}$ & $\mu f_{n}$ \\
\hline Lubrication & $\frac{12 \pi L \eta(\Omega-2 \dot{\psi}) r}{\left[h^{2}-r^{2}\right]\left[2 h^{2}+r^{2}\right]}$ & $-\frac{12 \pi L \eta R_{2}^{3} \dot{r}}{\left[h^{2}-r^{2}\right]^{3 / 2}}$ \\
\hline
\end{tabular}

Table 1: Table summarizing normal and tangential forces for different models. The fluid lubrication model (bottom row) is discussed in section 4.3.3.

which adds another nonlinearity into the system.

Hence, even the simplest contact model (van der Heijden in the top row of the table), which is linear in that $F_{n}=k_{c} \delta$, should have enough non-linearities in $x$ and $y$ (due to $\delta$ and $\psi$ ) to generate subharmonics. The other models (second to add tangential forces (friction/rubbing) and nonlinearities in $\delta$, which will obviously alter the dynamics, but it is not clear that they are needed to reproduce the key phenomenon we are after. Numerical simulations of these models will be used to shed light on this.

\subsubsection{Fluid/solid interaction}

We model the oil film between the rotor and stator using the lubrication approximation (gap size $\left.h \ll R_{1}, R_{2}\right)$. The pressure field $p(x, y)$ depends on the location $r$ and speed $\dot{r}$ of the rotor. The local gap between the rotor and stator $w(\theta, t)$ is a particularly important variable. 


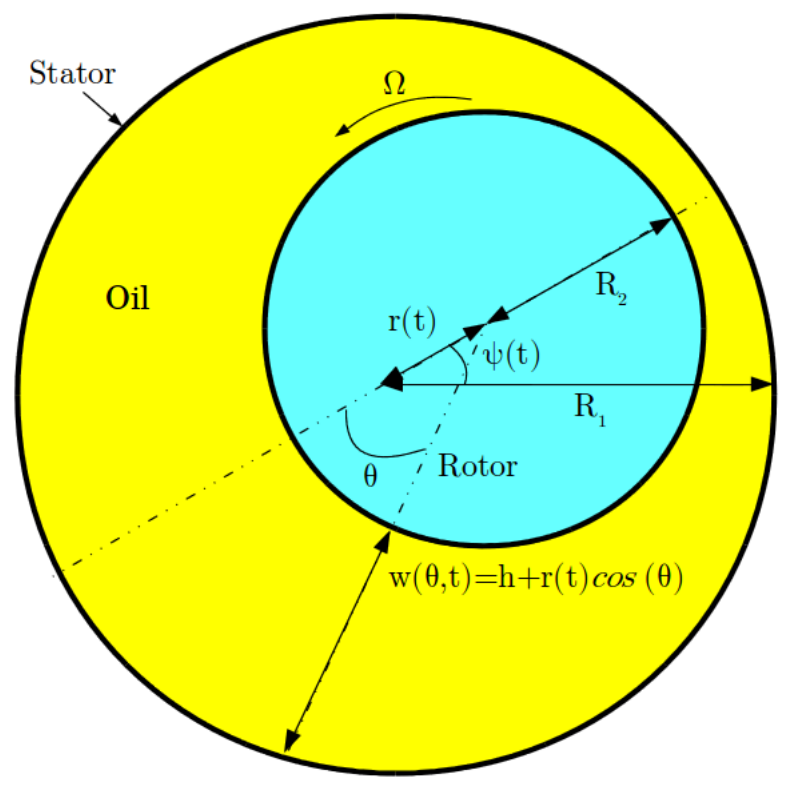

Figure 21: A model for an oil journal bearing

Consider the setup for the oil in a journal bearing as shown Fig. 21

The width of the gap, which we will define as $w(\theta, t)$ is assumed small relative to $R_{1}$, in the sense that $h \ll R_{1}$ where $h=R_{1}-R_{2}$; typical values are around 0.01 . This allows us to approximate

$$
w(\theta, t) \approx h+r(t) \cos \theta .
$$

Typically the Reynolds numbers for these flows will satisfy $R e \leq 1$, which allows us to neglect intertia and use a Stokes flow model; this is further simplified using the thin gap width by the lubrication approximation. Assuming flow is homogenous in the normal $z$-direction, suitable for a long rotor, we can use Reynolds' equation for lubrication flows:

$$
\frac{\partial}{\partial \theta}\left(\frac{w^{3}}{\eta R_{2}{ }^{2}} \frac{\partial p}{\partial \theta}\right)=6 \Omega \frac{\partial w}{\partial \theta}+12 \frac{\partial w}{\partial t} .
$$

Given the above form for $h(\theta, t)$ and periodicity in $\theta$, one can solve for the pressure $p$ :

$$
p=p_{0}+6 \mu R_{2}{ }^{2}\left(\frac{(\Omega-2 \dot{\psi})(2 h+r \cos \theta) r \sin \theta}{\left(2 h^{2}+r^{2}\right)(h+r \cos \theta)^{2}}+\frac{\dot{r}}{r}\left(\frac{h^{2}}{(h+r \cos \theta)^{2}}-\frac{h^{2}}{(h+r)^{2}}\right)\right) .
$$

The resulting forces (per spanwise unit length) in the directions $\theta=0, \theta=\frac{\pi}{2}$ from integrating across the full film are given by

$$
F_{n}=-\frac{12 \pi \eta R_{2}^{3} \dot{r}}{\left(h^{2}-r^{2}\right)^{3 / 2}}
$$




$$
F_{t}=\frac{12 \pi \eta R_{2}{ }^{3} r(\omega-2 \dot{\psi})}{\left(2 h^{2}+r^{2}\right)\left(h^{2}-r^{2}\right)^{1 / 2}} .
$$

However, this model is somewhat flawed, in that it fails to account for cavitation, where the above expression for pressure becomes negative. This suggests that the model breaks down, though crudely one could instead set the pressure to 0 in any region where it would otherwise be negative (i.e. treating these regions as 'fluid free'). For details, see [39].

After some algebra, we obtain the following 'Reynolds' equation for the pressure:

$$
\begin{gathered}
\frac{\partial}{\partial \theta}\left(\frac{w^{3}}{\eta R_{2}{ }^{2}} \frac{\partial p}{\partial \theta}\right)=6 \Omega \frac{\partial w}{\partial \theta}+12 \frac{\partial w}{\partial t} \\
F_{n}=\frac{12 \pi L \eta(\Omega-2 \dot{\psi}) r}{\left[h^{2}-r^{2}\right]\left[2 h^{2}+r^{2}\right]} \\
F_{t}=-\frac{12 \pi L \eta R_{2}^{3} \dot{r}}{\left[h^{2}-r^{2}\right]^{3 / 2}}
\end{gathered}
$$

These are given in the bottom row of table 1 .

\subsubsection{Simulation model}

For our simulations we consider the following set of differential equations

$$
\begin{aligned}
& m \ddot{x}-c_{1} m \Omega \dot{y}+c_{2} \dot{x}+k_{1} x+k_{2} y=m \varepsilon \Omega^{2} \cos \Omega t+F_{x} \\
& m \ddot{y}+c_{1} m \Omega \dot{x}+c_{2} \dot{y}-k_{2} x+k_{1} y=m \varepsilon \Omega^{2} \sin \Omega t+F_{y}
\end{aligned}
$$

where the parameters are as follows:

$$
\begin{array}{lll}
m & \ldots & \text { mass } \\
\Omega & \ldots & \text { driving frequency } \\
\varepsilon & \ldots & \text { eccentricity } \\
c_{1} & \ldots & \text { constant for gyroscopic terms, default to } 0 \\
c_{2} & \ldots & \text { damping } \\
k_{1} & \ldots & \text { stiffness } \\
k_{2} & \ldots & \text { stiffness cross coupling, default to } 0 .
\end{array}
$$

We can express $F_{x}, F_{y}$ further as

$$
\begin{aligned}
& F_{x}=-F_{n} \cos \psi+F_{t} \sin \psi \\
& F_{y}=-F_{n} \sin \psi-F_{t} \cos \psi
\end{aligned}
$$


with

$$
\begin{aligned}
& \sin \psi=\frac{y-\varepsilon \sin \Omega t}{r} \\
& \cos \psi=\frac{x-\varepsilon \cos \Omega t}{r}
\end{aligned}
$$

and $F_{n}, F_{t}$ being chosen depending on the model (see Table 1 ).

We have written a Python code provided, in which we have implemented the general system of differential equations (22), rewritten as a system of first order DE, with $F_{x}, F_{y}$ and possible additional constants given for each model individually. We provide more information about the supplied code in the subsection ??.

\subsection{Simulation Results}

In this subsection, we include results of two models, van der Heijden and the Lubrication one, to illustrate creation of 'humps' in the spectrum and development of sub-harmonics. We need to stress that even though our literature review revealed that the relationship between spectra of displacements and current are not linked linearly, we are unsure what their exact relationship is. The nature of their true dependence requires further investigation.

To be able to at least partially compare the models, we decided on a 'default set' of parameters which is

$$
\begin{array}{rr}
m=10, & \varepsilon=0.03 \\
c_{1}=0, & c_{2}=0.1 \\
k_{1}=10, & k_{2}=0 .
\end{array}
$$

This then gives us the natural frequency as

$$
\Omega_{\mathrm{nat}}=\sqrt{\frac{k_{1}}{m}}=1 .
$$

You might realize that the value for the gap parameter is missing in (23). This is due to the fact that as its size closely depends on the behaviour of the chosen model, the size of the gap needs to be varied to suit the studied model. We chose $h=0.05$ for the van der Heijden model and $h=0.3$ for the Lubrication model. Further more, the following additional parameter

$$
k_{c}=100
$$

was used for the van der Heijden model and

$$
\eta=10^{-5}, \quad R_{2}=0.1 \cdot h=0.03
$$

were used for the Lubrication model. 


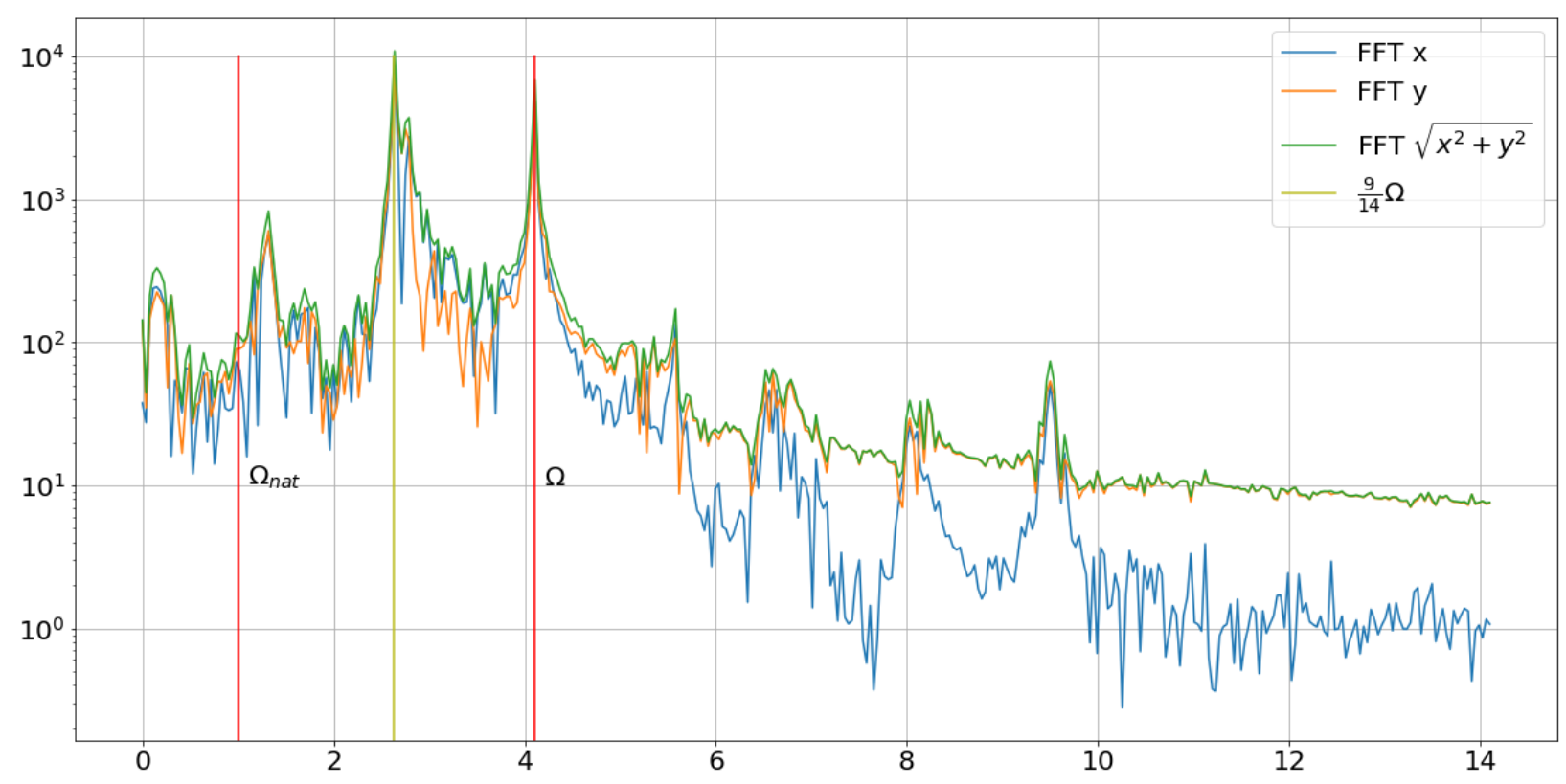

Figure 22: Evidence of sub-harmonics for van der Heijden model with default set of parameters (23) with lower damping $c_{2}=0.05$ and $\Omega=4.1$. Especially note that $\varepsilon=0.03$. The position of the most prominent sub-harmonic is indicated. Compare with Fig. 23 to see the effect of varying the eccentricity $\varepsilon$.

\subsection{1 van der Heijden model}

First presented model is the easiest non-trivial model - van der Heijden, which only considers the normal force and ignores any friction. However, even this model is capable of producing sub-harmonics (see Figure 22 and 23).

We found out that varying the eccentricity $\varepsilon$ while keeping the other parameters fixed (we chose $\Omega=4.1$ ) changes the nature of the graph (it is 'messy' for some values of $\varepsilon$ and suddenly calms for others) as well as the position of the sub-harmonic with the largest amplitude.

Some examples of both the stationary phase portraits and phase portraits in rotating frame (where the driving frequency has been negated) can be found on Figure 24. See Figure 25 for a waterfall plot exploring the relationship of spectra and the driving frequency $\Omega$.

\subsubsection{Lubrication model}

The second presented model is the Lubrication model as we believe that it facilitates the formation of humps (Figures 28 and 29).

Examples of phase portraits can be found on Figure 26 (we used same parameters as in 


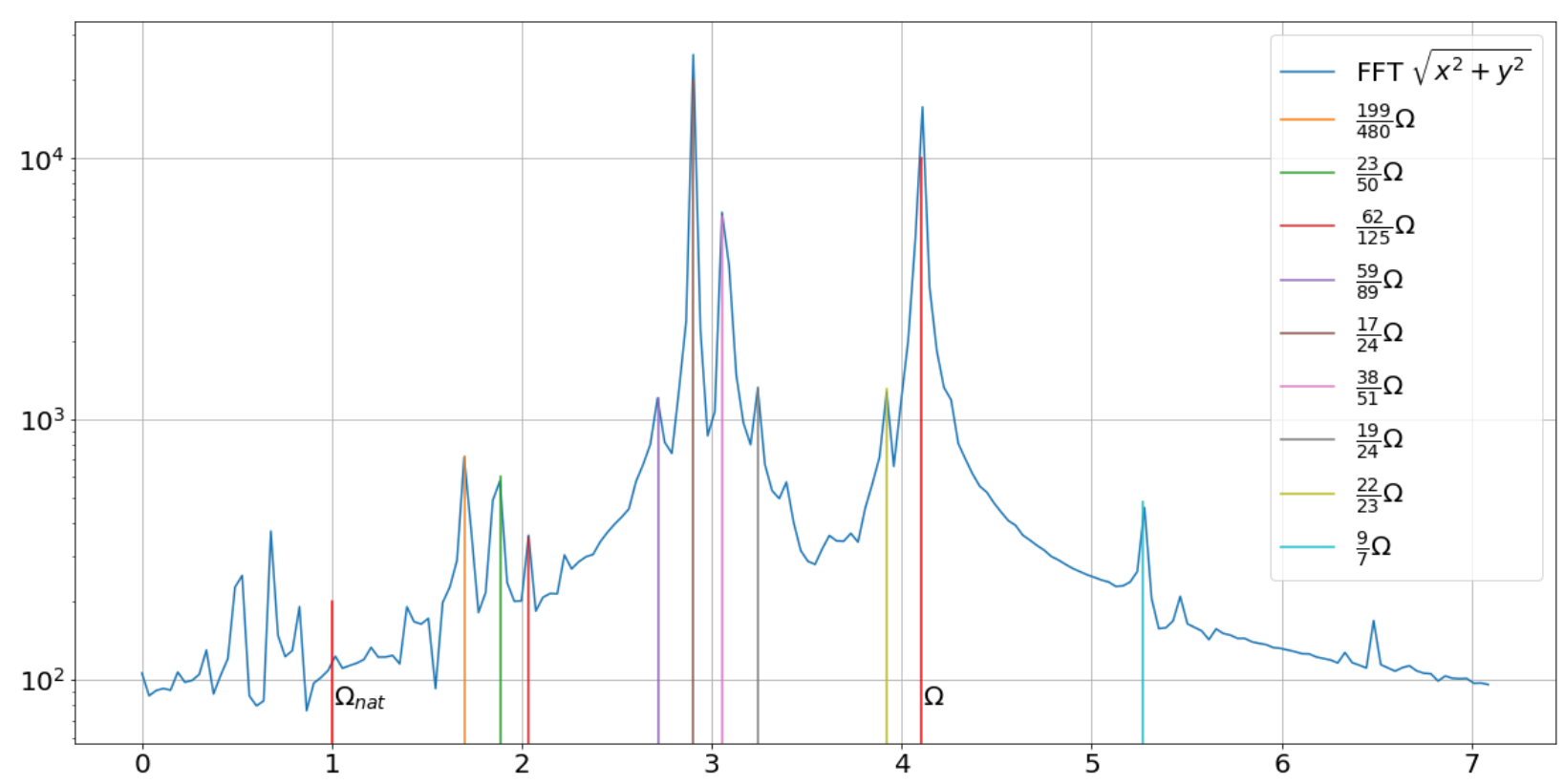

Figure 23: Graph of spectrum of frequencies for the van der Heijden model with default set of parameters (23) with lower damping $c_{2}=0.05$ and larger eccentricity $\varepsilon=0.0525$ and for $\Omega=4.1$. The position of most sub-harmonics was approximated.

Figure 24 with the only exception of the different gap $h=0.3$ ). Figure 27 then shows a waterfall plot for the Lubrication model.

As Figure 27 clearly shows, this model most likely does not produce sub-harmonics (at least for our set of parameters (23)). It does, however, produce a stable hump-like structures beyond the rotor speed $\Omega$. Note that this is highly sensitive to the particular rotor speeds. For some rotor speeds $\Omega$ there appears a significant 'dip' beyond the main resonance peak, but for others it appear a s significant hump. One possible explanation of this is that it is that the lubrication produces an effective nonlinear damping, which is highly dependent on the ratio of the drive frequency $\Omega$ and the natural frequency $\Omega_{\text {nat }}$. This effect seems unaffected by either an increase in eccentricity (see Figure 28) or in damping (Figure 29). Increasing the eccentricity increases the amplitude of the hump, whereas an increase in damping decreases the hump and changes the importance of $\Omega_{\text {nat }}$ and $\Omega$. 

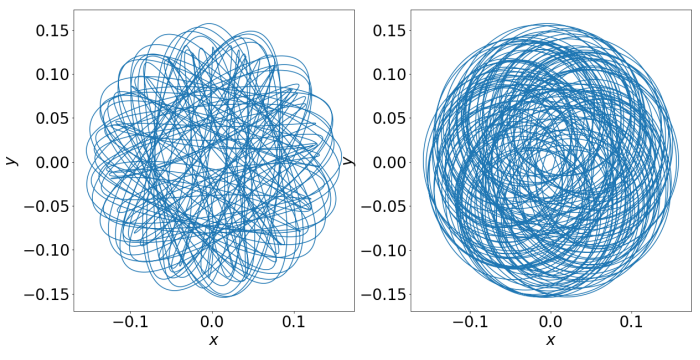

(a) $\Omega=1.15$

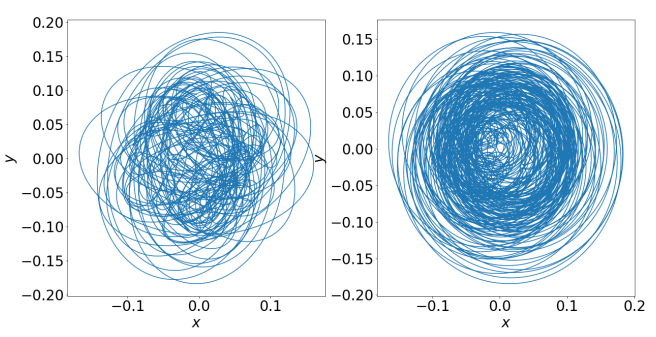

(c) $\Omega=3$
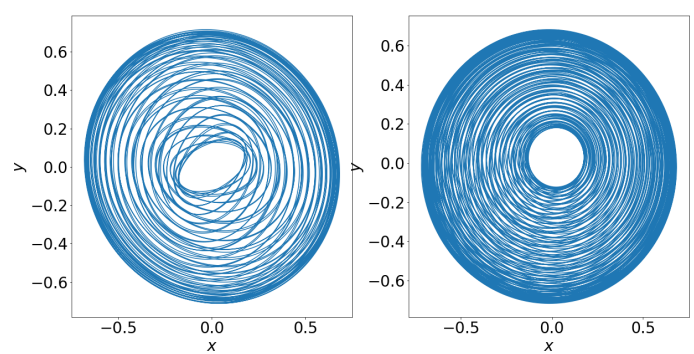

(b) $\Omega=2.1$

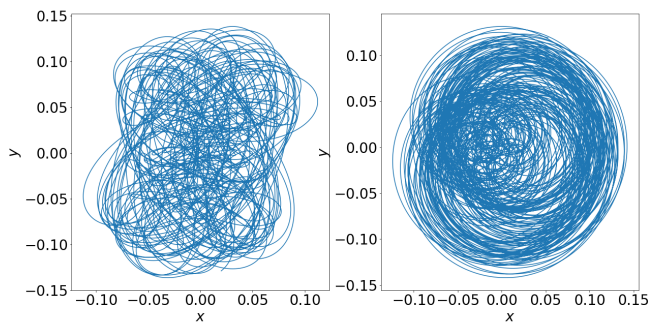

(d) $\Omega=4.1$

Figure 24: Phase portraits in stationary and rotating frames (on the left and right respectively) for 4 different values of the driving frequency $\Omega$.

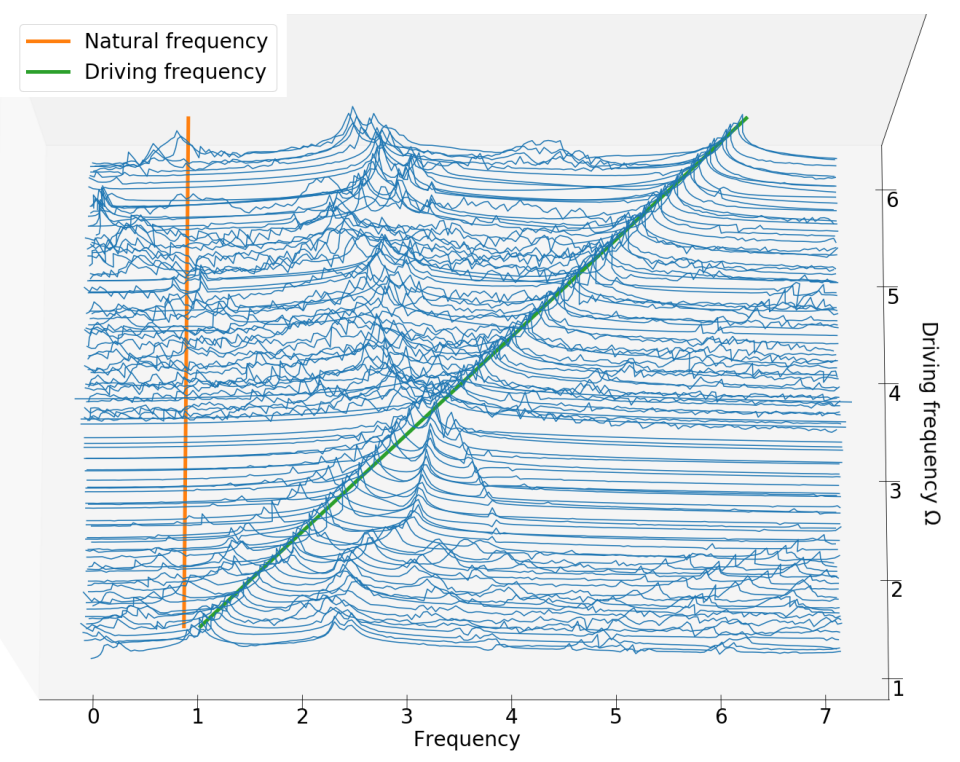

Figure 25: Waterfall plot of spectral dependence on the driving frequency $\Omega$ for the van der Heijden model. 

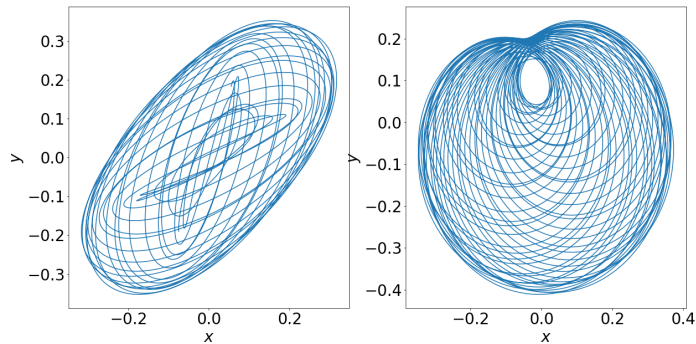

(a) $\Omega=1.15$
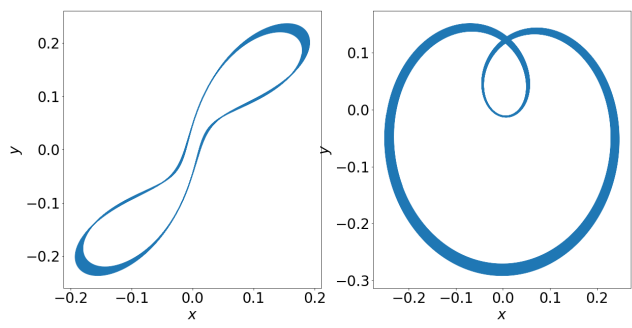

(c) $\Omega=3$
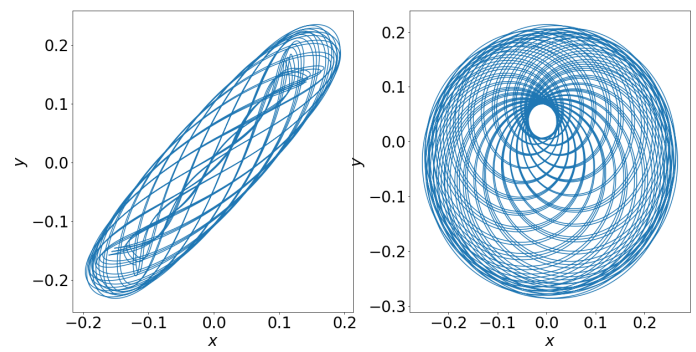

(b) $\Omega=2.1$
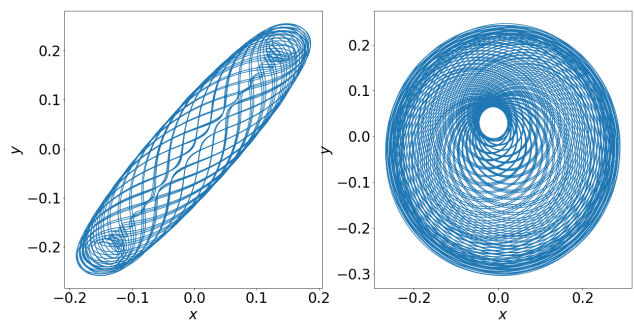

(d) $\Omega=4.1$

Figure 26: Phase portraits in stationary and rotating frames (on the left and right respectively) for 4 different values of the driving frequency $\Omega$.

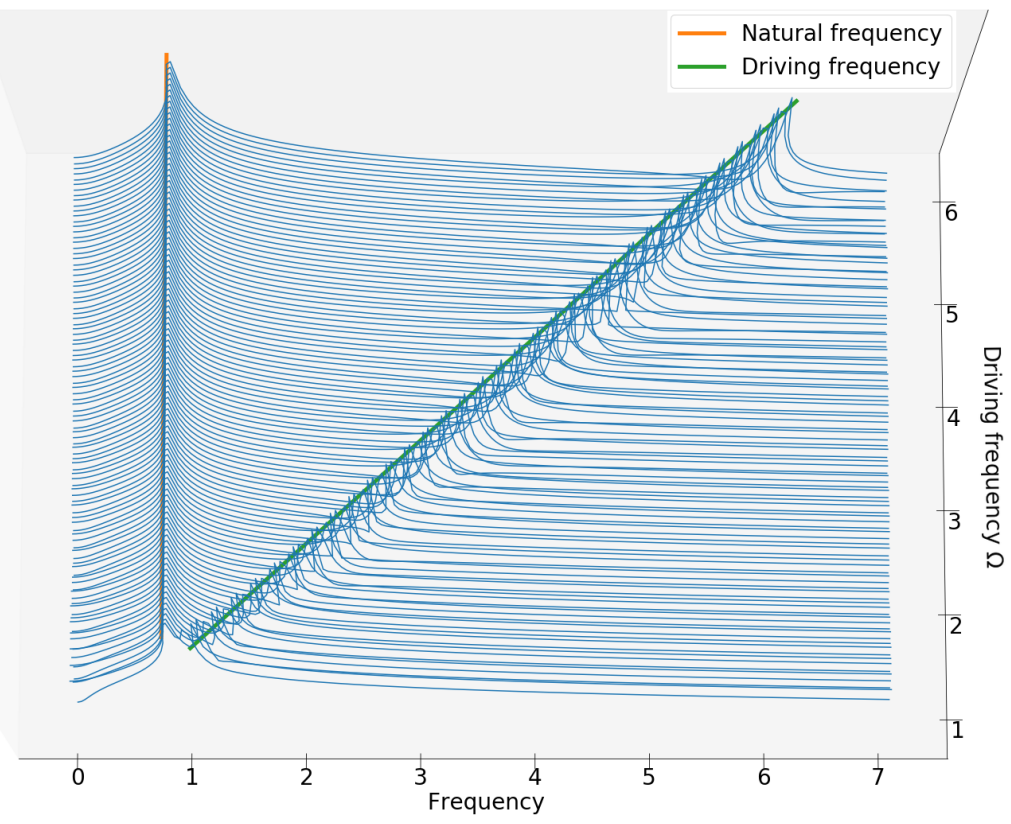

Figure 27: Waterfall plot of FFT for Lubrication model 


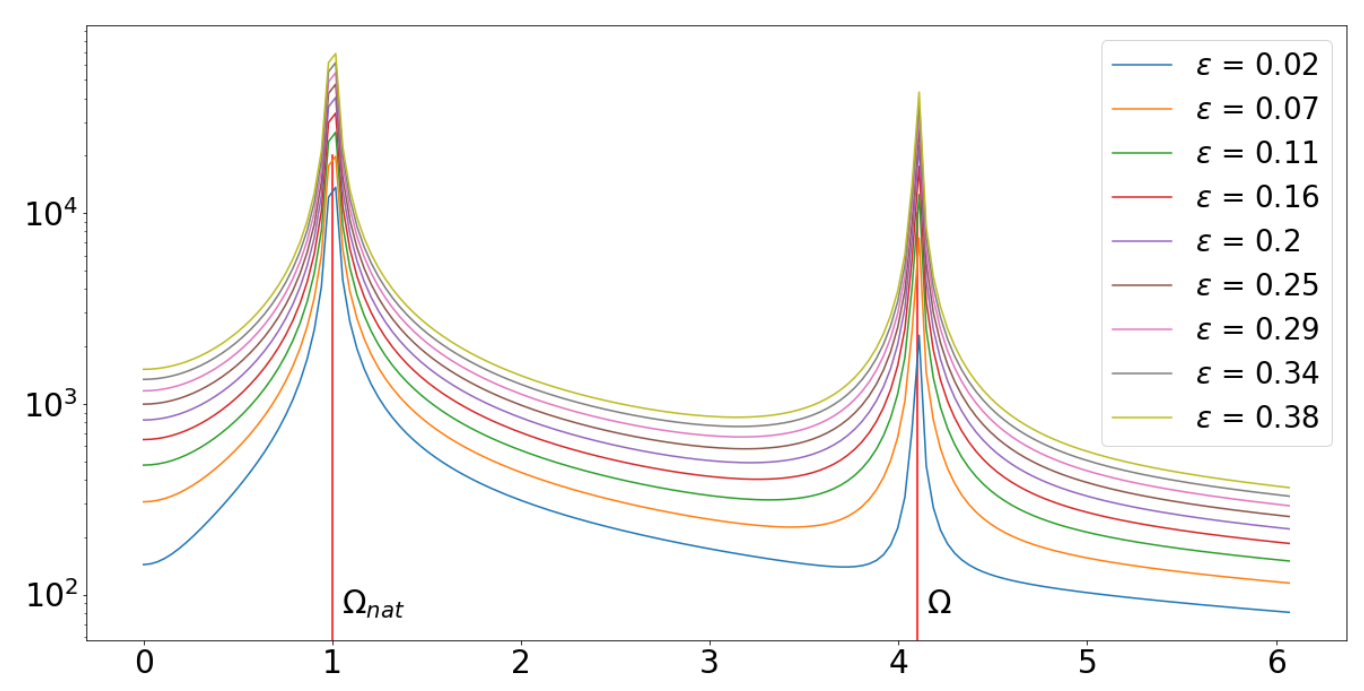

Figure 28: Demonstration of increasing the eccentricity for the Lubrication model. The eccentricity is varied from $\varepsilon=0.02$ to $\varepsilon=0.38$ with all the other parameters kept constant at their default values and with $\Omega=4$.1. Only the FFT of $\sqrt{x^{2}+y^{2}}$ is plotted for simplicity
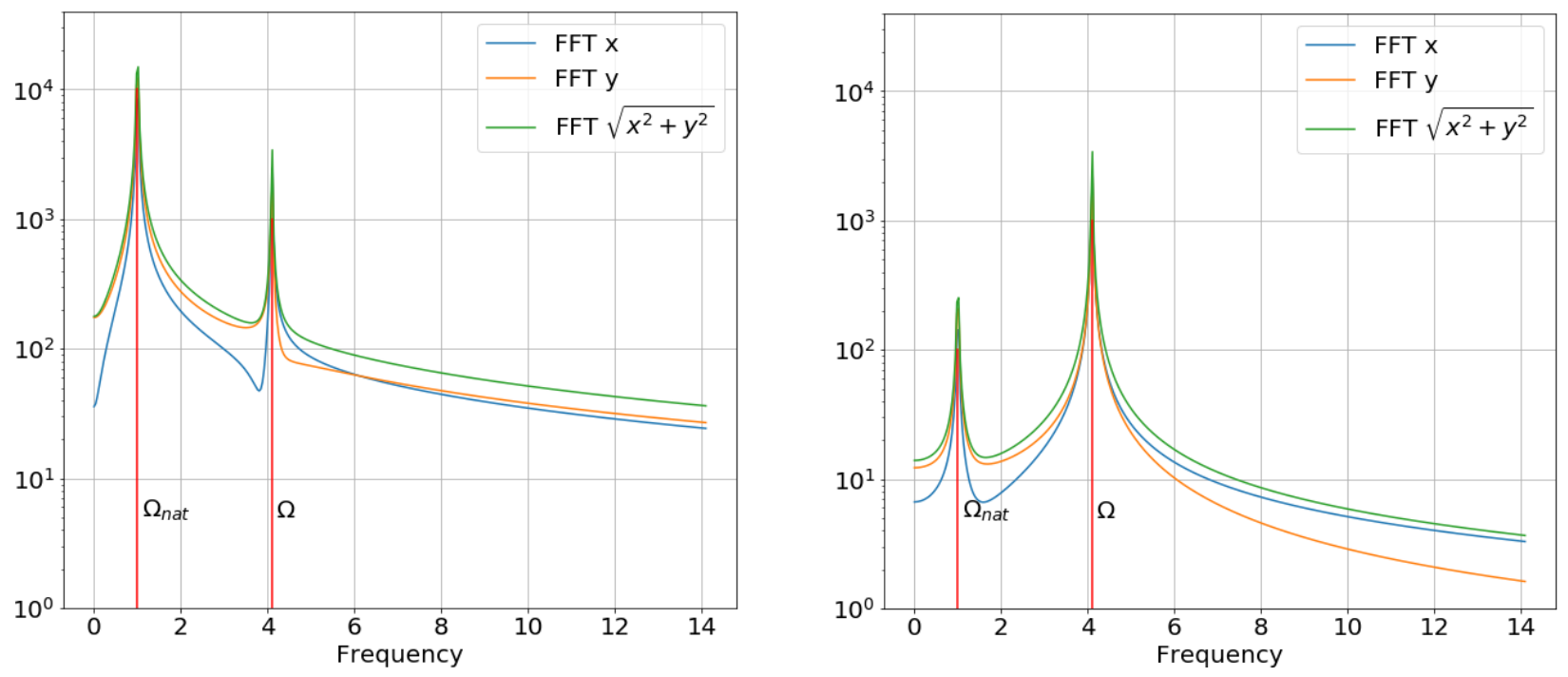

Figure 29: Demonstration of the effect of increased damping on the Lubrication model. For small damping ( $c_{2}=0.01$, on the left), the natural frequency is dominant in the spectrum. With increasing damping $\left(c_{2}=0.1\right.$, on the right), the driving frequency takes over and the whole 'hump' in between decreases. All other parameters were kept the same (default) set (see 23) with the driving frequency $\Omega=4.1$. 


\section{$5 \quad$ Main Findings and Recommendations}

1. Methods for automatic detection of spectral anomalies:

- We have implemented a routine for the automatic detection of humps in the spectral data. This can be used to identify regions of raised activity compared to a baseline

- So far we have tested this only a limited range of data, but it seems to be quite robust. One weakness is that the algorithm is currently only able to identify the most dominant hump in the data. But this could in principle be extended to detect further humps and its sensitivity to various parameters investigated.

Recommendation $\mathbf{1}$ Extend the hump detection algorithm to detect
multiple humps in the data. Test both the current and extended algorithm
on more data sets. In particular seek to optimise the threshold for hump
identification and the various ways of measuring maxima, derivatives and
convolutions as set out in Sec. 2.3 above.

2. Methods for identiying the presence of amplitude and phase modulation:

- We found evidence in many of the data sets provided of subsidiary peaks in the data that are regularly spaced around the fundamental frequency in the output. We have elucidated the contrasting hallmarks of amplitude and phase modulation, which may be caused by mode coupling or weak nonlinearity.

- We have provided a code that uses the Hilbert transform to perform automatic idenification of amplitude modulation and to reconstruct the signal using the dominant frequences so identified. The results are successful in identifying the origins of some of the subsidiary peaks in various data sets.

Recommendation 2 Extend the Hilbert transform algorithm to deal with phase modulation in addition to amplitude modulation:

3. Nonstationarity and additional peaks:

- We have noted that some effects, including the broadening of peaks can be an artifact of using a finite window of signal (typically 6.5s) to characterise the performance of a rotating machine. We note that these can be caused by low frequency modulation of fundamental frequeny peaks present in the system. It is possible that apparent humps in the data may be resolved by computing signal over longer times

Page 40 of 48 
- It is also the case that nonlinearity and imperfection within any rotor is likely to lead to nonstationarity. In particular, there is plenty of evidence from the literature that bearing distress or other rub or contacts can give rist to chaotic dynamics. By its very nature, chaotic dynamics is nonstationary and leads to broad-band components within the spectrum.

Recommendation 3 Perform a more systematic study on some of the more problematic spectra to determine the extent of nonstationarity within the signal and the effect of taking both longer time slices and compare the results of different time slices for evidence of artifical peak broadening and/or chaotic dynamics.

4. Mathematical models of different rub and contact effects in eccecntric rotors:

- Through a careful synthesis of the existing literature we have produced a suite of mathematical modes in python that can be run to produce synthetic spectra, orbit diagrams and waterfall plots of spectrum with rotor speed, that are consistent with those seen in the literature. These assume the simplest possible rotor model, but implement a range of different nonlinear rub, contact and lubrication nonlinearities.

- Using realistic generic parameter values, we found that we could reproduce the existence of additional subharmonic peaks and hump-like portions of spectrum. We also found evidence of humps or dips in higher frequency components, beyond the rotor frequency.

- One item of confusion that requires futher clarification is that during the study group we were told that for the data we were given the rotor's resonance frequency was likely to be much higher than the rotor speed (i.e. that these rotors are sub-critical). However, on careful insight of the literature, all of the interesting dyanmics that has been reported as a result of rub or contact appears to be for super-critical rotors, that is for where the rotor speed is beyond the first natural vibration frequency of the rotod.

- It should be stressed that during the study group we had limited time to test and run the models we implemented. Some work was done post study-group, but in general we found that the results are highly sensitive to parameter values, especially eccentricity, damping, and various parameters that characterise the nonlinearity. Reasonable parameter values were only sought for the van der Heijden an lubrication models. The others essentially remain untested.

- Our results followed most studies in the literature, in which we considered the rotor speed $\Omega$ to be the main bifurcation parameter, for which we consider the 
dynamic effects of its variation. However, what would appear interesting from a Faraday Predictive perspective would be to use a damage or distress parameter as a bifurcation parameter.

Recommendation 4 Perform a more systematic parametric study of one or more of the nonlinear rotor models. It would be good to have some test data for a particular machine and then attempt to derive and fit parameters of that machine to a nonlinear dynamics model. Then we could consider a systematic study that attempted to explain the evolution of damage over time. Ultimately this is what we understand is Faraday Predictive's core aim.

5. Linking the spectral analysis and the simulation model:

- A goal was identified during the study group to use the spectral analysis algorithms we have developed on data generated from the simulation models. This was unrealistic within the four days of work time that was available. This remains a valid and pressing line of investigation.

Recommendation 5 Further test and perfect the spectral analysis
software by testing on a simulation model for which we know the cause
of the nonlineartiy or signal anomaly. This should enable testing of hy-
potheses about which effects cause which kind of anomaly and how to
develop a reliable test for it.

6. A possible indicator of bearing distress:

- We have developed an understanding of how subharminic peaks of different frequencies at a rational ratio $p / q$ of the fundamental drive frequency can arise where $q>p$ is typically a prime number. These represent frequency-locking due to nonlinear effects where a natural frequency of vibration of a nonlienar component of the system is close to a rational ratio $p / q$ of the fundamental. The theory of Arnol'd tongues shows that such behaviour should become more pronounced the stronger the level of nonlinearity. Such orbits are typically characterised by being perfect limit cycles with $p$ loops, when viewed in a rotating frame.

- These findings confirm and help explain the combined experimental and numerical results of Ma, Muszynska and Groll \& Ewins in the literature [31, 7, 22]. They show that in the presence of a rub-type nonlinearity with a stator for a fixed level of eccentricity, then a succession of different $p / q$ orbits are excited as the rotor speed is increased. 
- It is interesting to note that the suggest there could be a way of indicating the level of bearing distress Bearing distress occurs as a result of contact between the rotor (journal) and the stator (bearing). Contact forces increase with penetration between the two surfaces, determined by the eccentricity of the rotor.

- One hypothesis is that increased contact forces give rise to more and more complex quasiperiodic vibration patterns ( $p$-loops with increasing $p$ ), these appear as subharmonic frequencies in the spectrum at rational fractions of the main rotor speed.

Recommendation 6 Further understanding of this distress hypothesis should be sought by performing a bifurcation anlaysis of the Arnol'd toungues of a simple rotor dynamic model such as that analysed here. Also ascertain whether humps in the spectrum can represent chaotic dynamics which is typically observed outside of the tongues.

\section{References}

[1] F. Immovilli, A. Bellini, R. Rubini, and C. Tassoni. Diagnosis of bearing faults in induction machines by vibration or current signals: A critical comparison. IEEE Transactions on Industry Applications, 46(4):1350-1359, 2010.

[2] R. Ebrahimi, M. Ghayour, and H.M. Khanlo. Nonlinear dynamic analysis and experimental verification of a magnetically supported flexible rotor system with auxiliary bearings. Mechanism and Machine Theory, 121:545-562, 2018.

[3] Loï Salles, Bernard Staples, Norbert Hoffmann, and Christoph Schwingshackl. Continuation techniques for analysis of whole aeroengine dynamics with imperfect bifurcations and isolated solutions. Nonlinear Dynamics, 86(3):1897-1911, nov 2016.

[4] C. Wang, D. Zhang, Y. Ma, Z. Liang, and J. Hong. Theoretical and experimental investigation on the sudden unbalance and rub-impact in rotor system caused by blade off. Mechanical Systems and Signal Processing, 76-77:111-135, 2016.

[5] N.N. Verichev. Chaotic torsional vibration of imbalanced shaft driven by a limited power supply. Journal of Sound and Vibration, 331(2):384-393, 2012.

[6] A. Zilli, R.J. Williams, and D.J. Ewins. Nonlinear Dynamics of a Simplified Model of an Overhung Rotor Subjected to Intermittent Annular Rubs. Journal of Engineering for Gas Turbines and Power, 137(6):065001, jun 2015.

[7] A. Muszynska. Rotordynamics. Taylor \& Francis, London, 2005. 
[8] M.F. Abdul Azeez and A.F. Vakakis. Numerical and experimental analysis of a continuous overhung rotor undergoing vibro-impacts. International Journal of Non-Linear Mechanics, 34(3):415-435, 1998.

[9] A. Boyaci. Numerical Continuation Applied to Nonlinear Rotor Dynamics. Procedia IUTAM, 19:255-265, 2016.

[10] F.F. Ehrich. The Dynamic Stability of Rotor/Stator Radial Rubs in Rotating Machinery. Journal of Engineering for Industry, 91(4):1025, 1969.

[11] H. Bleuler, M. Cole, P. Keogh, R. Larsonneur, E. Maslen, Y. Okada, G. Schweitzer, A. Traxler, G. Schweitzer, and E.H. Maslen. Magnetic bearings: theory, design, and application to rotating machinery. Springer Science \& Business Media, 2009.

[12] S. E. Khadem, M. Shahgholi, and S. A.A. Hosseini. Primary resonances of a nonlinear in-extensional rotating shaft. Mechanism and Machine Theory, 45(8):1067-1081, 2010.

[13] J. Łuczko. A geometrically non-linear model of rotating shafts with internal resonance and self-excited vibration. Journal of Sound and Vibration, 255(3):433-456, 2002.

[14] Nizar Ferjaoui, Sami Naimi, and Mnaour Chouchane. Bifurcation analysis of a flexible balanced cracked rotorbearing system. Comptes Rendus - Mecanique, 344(9):661-671, 2016.

[15] K. Green, A.R. Champneys, and N.J. Lieven. Bifurcation analysis of an automatic dynamic balancing mechanism for eccentric rotors. Journal of Sound and Vibration, 291(3-5):861-881, 2006.

[16] D.J. Rodrigues, A.R. Champneys, M.I Friswell, and Wilson R.E. Experimental investigation of a single-plane automatic balancing. J. Sound Vib., 330:385-403, 2011.

[17] N. van de Wouw, H. Nijmeijer, and N. MihajloviĆ. On the Coupling Between Torsional and Lateral Vibrations in a Rotor Dynamic System With Set-Valued Friction. In Volume 5: 6th International Conference on Multibody Systems, Nonlinear Dynamics, and Control, Parts A, B, and C, pages 213-222. ASME, 2007.

[18] G.H.M. van der Heijden. Mode-locking in nonlinear rotordynamics. Journal of Nonlinear Science, 5:257-283, 1995.

[19] A.P. Christoforou and A.S. Yigit. Fully coupled vibrations of actively controlled drillstrings. Journal of Sound and Vibration, 267(5):1029-1045, nov 2003.

[20] P. Varney and I. Green. Nonlinear phenomena, bifurcations, and routes to chaos in an asymmetrically supported rotor-stator contact system. Journal of Sound and Vibration, $336: 207-226,2015$. 
[21] F.K. Choy and J. Padovan. Non-linear transient analysis of rotor-casing rub events. Journal of Sound and Vibration, 113(3):529-545, mar 1987.

[22] G.V. Groll and D.J. Ewins. A mechanism of low subharmonic response in rotor/stator contact-measurements and simulations. Transactions of the ASME: Journal of Vibration and Acoustics, 124(July 2002):350-358, 2002.

[23] W. Qin, G. Chen, and G. Meng. Nonlinear responses of a rub-impact overhung rotor. Chaos, Solitons \& Fractals, 19(5):1161-1172, mar 2004.

[24] W.-M. Zhang and G. Meng. Stability, bifurcation and chaos of a high-speed rub-impact rotor system in MEMS. Sensors and Actuators A: Physical, 127(1):163-178, feb 2006.

[25] J. Isaksson and A. Frid. Analysis of dynamical behaviour of a rotor with deflection limiters. Acta Mechanica, 133:1-11, 1999.

[26] M. Karlberg and J.O. Aidanpää. Investigation of an unbalanced rotor system with bearing clearance and stabilising rods. Chaos, Solitons $\&$ Fractals, 20(2):363-374, apr 2004.

[27] S. Edwards, A.W. Lees, and M.I. Friswell. The Influence of Torsion on Rotor/Stator Contact in Rotating Machinery. Journal of Sound and Vibration, 225(4):767-778, 1999.

[28] N. Vlajic, A.R. Champneys, and S. Balachandran. Nonlinear dynamics of a Jeffcott rotor with torsional deformations and rotor-stator contact. Int. J. Nonlinear Mech., 92:102-110, 2017.

[29] K. Mora, C. Budd, P. Glendinning, and P. Keogh. Non-smooth Hopf-type bifurcations arising from impact-friction contact events in rotating machinery. Proc. Roy. Soc. Lond. A, 470:art. no. 20140490, 2014.

[30] A.D. Shaw, A.R. Champneys, and M.I. Friswell. Asynchronous partial contact motion due to internal resonance in multiple degree-of-freedom rotordynamics. Proceedings of the Royal Society A: Mathematical, Physical and Engineering Science, 472(2192):20160303, aug 2016.

[31] H. Ma, Q. Zhao, X. Zhao, Q. Han, and B. Wen. Dynamic characteristics analysis of a rotor-stator system under different rubbing forms. Applied Mathematical Modelling, 39:2392-2408, 2015.

[32] Chaotic responses of unbalanced rotor-bearing stator systems with looseness or rubs.

[33] C. Bai, H. Zhang, and Q. Xu. Subharmonic resonance of a symmetric ball bearing-rotor system. International Journal of Non-Linear Mechanics, 50:1-10, 2013. 
[34] E. Malcolm and P. Leader. Understanding journal bearings, 2001.

[35] H. Boucherit, B.S. Benyebka, and L Mustapha. The effect solid particle lubricant contamination on the dynamic behavior of compliant journal bearings. Lubrication Science, 29:425-439, 2017.

[36] Lubrication and roughness. In: Tribology for Engineers by Davim, J.P.

[37] M.I. Friswell, J.E.T. Penny, S.D. Garvey, and A.W. Lees. Dynamics of Rotating Machines. Cambridge University Press, 2010.

[38] C. Hua, Z. Rao, N. Ta, and Z. Zhu. Nonlinear dynamics of rub-impact on a rotorrubber bearing system with the stribeck friction model. Journal of Mechanical Science and Technology, 29(8):3109-3119, 2015.

[39] P. Flores, H.M. Lankarani, J. Ambrósio, and J.C.P. Claro. Modelling lubricated revolute joints in multibody mechanical systems. Proceedings of the Institution of Mechanical Engineers, Part K: Journal of Multi-body Dynamics, 218(4):183-190, 2004.

\section{A Supplied Code}

\section{A.1 Spectral analysis}

The code described below are available on dropbox https://www.dropbox.com/1/scl/AABDP8s-SohfsLmiu3iTzHYXDayPPrOVxKU in the CODE folder.

hump_detection.py The program hump_detection.py is in the subfolder HUMP. Together with the 2 input files it uses as examples. These can be changed line 22 and 23 of the program.

The program output 3 figures:

1. The absolute value of the Fourier Transform of the residual current and the anomalous spectrum (1)

2. The anomalous spectrum (1) and its low-pass filtered version.

3. The anomalous spectrum (1) and its low-pass filtered version with 3 vertical bars indicating the left, centre and right of the detected hump.

The program also outputs on the console, the $L^{2}$ norm of the filtered anomalous spectrum (with and without the logarithm) as well as the $L^{2}$ restricted to the detected hump. 
sawtooth_modulation.py The program sawtooth_modulation.py is in the Spectrum subfolder. It generates several time-phase modulation time sequences and display their spectrum. This include several sawtooth phase modulations.

fm_spectrum_dbl.py The program fm_spectrum_dbl.py is in the Spectrum subfolder and it generates figures of several phase modulation spectra:

1. $\log |\mathrm{FFT}|$ of $\sin (2 \pi 60 t+3 \sin (2 \pi 6 t))$

2. Sawtooth function.

3. $\log |\mathrm{FFT}|$ of $\sin (2 \pi 60 t+3 \operatorname{SawTooth}(2 \pi 60 / 16 t))$

4. $\log |\mathrm{FFT}|$ of $\sin (2 \pi 60 t+2 \operatorname{SawTooth}(2 \pi 60 / 16 t))$

5. $\log |\mathrm{FFT}|$ of $\sin (2 \pi 60 t+5 \operatorname{SawTooth}(2 \pi 60 / 16 t)$

6. $\log |\mathrm{FFT}|$ of $\sin (2 \pi 50 t+\sin (2 \pi 12.5 t)+6 \sin (2 \pi 6.25 t)$

7. $|\mathrm{FFT}|$ of $\sin (2 \pi 50 t+\sin (2 \pi 12.5 t)+6 \sin (2 \pi 6.25 t)$

AM_reconstruction_v2.py The program AM_reconstruction_v2.py is in the Spectrum folder. It analyses the time series GenA-1_ia.csv and extract its amplitude modulation. The main parameters one needs to adjust for different signals are initialised from line 50 and are:

- hmin : the threshold value to filter amplitude modulations.

- $\mathrm{fc}$ : the carrier frequency

- fs : the sampling frequency

- $k m a x$ : the largest frequency to be shown on figures

The program outputs on the console, the list of frequencies that were selected to reconstruct the amplitude modulation, as well as their amplitude. It also generates the following figures:

- The spectrum of the residual current.

- The spectrum of the extracted amplitude modulation.

- The filtered version of the extracted amplitude modulation .

- The reconstructed modulation amplitude.

- The first 10 cycles of the reconstructed modulation amplitude.

- The spectrum of reconstructed time series with the original one. 


\section{A.2 Rotordyanmics simulation}

The code described below is again available on the Dropbox https://www.dropbox.com/l/scl/AABDP8s-SohfsLmiu3iTzHYXDayPPrOVxKU

in the NonlinearDynamicsSimulation folder. It consists of one Jupyter Notebook written in Python 3 named Final_version.ipynb, which is heavily commented throughout. Short summary of the code is as follows, with the order following the order of cells in the notebook:

1. Import of necessary packages and simplifying some notations (such as pi $=$ math.pi)

2. Definition of the first order systems for two different solvers, see below

3. Definition of the time vector, time step and initial condition, which is used in the following cell

4. Choosing the desired model, setting of parameters. The default set of parameters (23) is provided as default

5. Choosing of a solver (fixed step vs variable step one), possibly choosing a method for the variable step solver. Actual solving and extraction of the solutions

6. Removal of initial part of the solution to discard transient period, definition of the solutions in a rotating frame. Plotting the phase portrait for both the fixed frame and the rotating frame

7. Calculating and plotting the FFT transform of the data

8. Plotting data as a waterfall plot 